\title{
ON A SOLUTION OF THE CAUCHY PROBLEM IN THE WEIGHTED SPACES OF BEURLING ULTRADISTRIBUTIONS
}

\author{
STEVAN PILIPOVIĆ, BOJAN PRANGOSKI AND DANIEL VELINOV
}

\begin{abstract}
Results of Da Prato and Sinestrari [6] on differential operators with non-dense domain but satisfying the Hille-Yosida condition, are applied in the setting of Beurling weighted spaces of ultradistributions $\mathcal{D}_{L^{p}}^{\prime(s)}((0, T) \times U)$, where $U$ is open and bounded set in $\mathbb{R}^{d}$. For this purpose, the new structural theorems were given for $\mathcal{D}_{L^{p}}^{(s)}((0, T) \times U)$. Then a class of the Cauchy problems in the general setting of such spaces of ultradistributions is analyzed.
\end{abstract}

0. Introduction. Da Prato and Sinestrari [6] have studied the Cauchy problem

$$
u^{\prime}(t)=A u(t)+f(t), \quad u(0)=u_{0},
$$

where $A$ is a closed operator in a Banach space $E$ with not necessarily dense domain in $E$ but satisfying the Hille-Yosida condition. Here, $u_{0} \in E$ and $f$ is the $E$-valued continuous or $L^{p}$-function on $[0, T]$. They have considered various classes of equations and types of solutions illustrating their theory. Regularity properties of the solutions are extended much later in [26].

Our aim in this paper is to extend the results of [6] for (0.1) to weighted Schwartz spaces of distributions and Beurling space of ultradistributions [9]-[11]. Since the weighted Schwartz space $\mathcal{D}_{L^{p}}^{\prime}$ ([28]) can be involved in this theory similarly to Beurling type spaces, and the second ones are more delicate, we focus our investigation on the Beurling case, more precisely to the space of ultradistributions $\left.\mathcal{D}_{L^{p}}^{(s)}((0, T) \times U)\right), U$ a bounded domain in $\mathbb{R}^{d}$ related to the Gevrey sequence $p !^{s}, s>1$ (see [24] for $U=\mathbb{R}^{d}$ ). In order to apply results

2010 AMS Mathematics subject classification. Primary 47D03, Secondary $35 \mathrm{~A} 01$.

Keywords and phrases. Cauchy problem, strict weak solution, $F$-weak solution.

Received by the editors on January 23, 2014. 
of [6] in this abstract setting, we study the topological structure of spaces $\mathcal{D}_{L^{p}, h}^{s}(U), p \in[1, \infty]$ (with a special analysis for $p=\infty$ ), the closures of $\mathcal{D}^{(s)}(U)$ in such spaces, corresponding projective limits, tensor products, their duals as well as a vector-valued version of such spaces. As a special result, we note that $\mathcal{D}_{L^{p}}^{(s)}(U)$ is nuclear for bounded $U$. Also, we have that all spaces $\mathcal{D}_{L^{p}}^{(s)}(U)$ are isomorphic to $\dot{\mathcal{B}}^{(s)}(U)$ for bounded $U$. Both assertions do not hold for $U=\mathbb{R}^{d}$. The main results of the paper are related to the structure of quoted spaces. Such preparatory results are needed for the formulation of the Cauchy problem in this abstract setting and for the application of results in [6].

In the second part of the paper we apply our results for the Cauchy problem with the infinitesimal generators satisfying the Hile-Yosida condition. In fact, we prove the following result.

Theorem 0.1. Let $A: D(A) \subseteq E \rightarrow E$ be a closed operator which satisfies the Hille-Yosida condition and $\mathbf{f} \in \mathcal{D}_{L^{p}}^{(s)}(0, T ; E)$. Then the equation $\mathbf{u}^{\prime}=A \mathbf{u}+\mathbf{f}$ always has a solution $\mathbf{u} \in \mathcal{D}_{L^{p}}^{(s)}(0, T ; E)$.

In other words, we solve (0.1) in the space of Banach valued ultradistributions $\mathcal{D}_{L^{p}}^{(s)}(0, T ; E)$, i.e.,

$$
\left\langle\mathbf{u}^{\prime}(t), \varphi(t)\right\rangle=A\langle\mathbf{u}(t), \varphi(t)\rangle+\langle\mathbf{f}(t), \varphi(t)\rangle, \quad \forall \varphi \in \mathcal{D}_{L^{q}}^{(s)}(0, T),
$$

where $A: D(A) \subseteq E \rightarrow E$ is closed operator which satisfies the HilleYosida condition

$$
\left\|(\lambda-\omega)^{k} R(\lambda: A)^{k}\right\| \leq C, \quad \text { for } \lambda>\omega, k \in \mathbb{Z}_{+} .
$$

Then, by using this result and the theory that we previously developed, we prove the following theorem.

Theorem 0.2. Let $U$ be a bounded domain in $\mathbb{R}^{d}$ with smooth boundary, (i.e., $\mathcal{C}^{\infty}$ boundary) and let $A\left(x, \partial_{x}\right)$ be a strongly elliptic operator of order $2 m$ on $U$. Then, for each $f \in \mathcal{D}_{L^{p}}^{(s)}((0, T) \times U)$, there exists a $u \in \mathcal{D}_{L^{p}}^{(s)}((0, T) \times U)$ such that

$$
u_{t}^{\prime}+A\left(x, \partial_{x}\right) u=f \quad \text { in } \mathcal{D}_{L^{p}}^{(s)}((0, T) \times U) .
$$


Theorem 0.2 is applicable to a variety of situations, among which is the heat equation $u_{t}^{\prime}-\Delta_{x} U=f$ in the ultradistribution space $\mathcal{D}_{L^{1}}^{\prime(s)}((0, T) \times U)$, for $f \in \mathcal{D}_{L^{1}}^{\prime(s)}((0, T) \times U)$, and $U$ is a bounded domain that has a smooth boundary. In fact, one only needs to put $A\left(x, \partial_{x}\right)=-\Delta_{x}$ in Theorem 0.1 , and observe that $-\Delta_{x}$ is a strongly elliptic operator of order 2 on $U$ (for the definition of the strongly elliptic operator see Definition 5.1).

Let us point out that the quoted theorem can be treated by the classical theory of $C_{0}$ analytic semigroups but our goal was to use our results with the Hile-Yosida condition and the results within the theory of ultradistributions. In this sense, the denseness of the infinitesimal generator (which holds) was not important in the presentation of our existence result in $\mathcal{D}_{L^{1}}^{\prime(s)}((0, T) \times U)$. Moreover, jointly with the previous, our goal was to present the solvability of an important class of equations which was not treated up until now within distribution and ultradistribution spaces over a bounded domain.

For the background material, we mention $[\mathbf{1 8}, \mathbf{2 0}, \mathbf{2 2}, \mathbf{2 3}, \mathbf{2 5}]$. The abstract Cauchy problem in the distribution case was vastly studied. It can be threaded by using local integrated semigroups. In fact, in [19], by Theorems 2.1.3-2.1.5 and Propositions 1.2.3 and 1.2.4, the operator $A$, which has a dense domain, is a generator of a local $n$-times integrated semigroup, which is a solution to the Cauchy problem. In the non dense case, Proposition 4.6 of [15] states the existence of a local $n$-times integrated semigroup $V(t)$ generated by $A$, which gives the solution to the Cauchy problem. Moreover, we give references for other approaches to the abstract Cauchy problem with non-densely defined $A$ through the convoluted, distribution or ultradistribution semigroups, $[1]-[5,7,13]-[17,19,21]$.

The paper is organized as follows.

The Banach space $\mathcal{D}_{L^{p}, h}^{(s)}(U)$ and its dual $\mathcal{D}_{L^{p}, h}^{(s)}(U)$ are explained in Section 1.

Section 2 is devoted to the Beurling type test spaces $\mathcal{D}_{L^{p}}^{(s)}(U)$ and their corresponding duals.

In Section 3, we consider the vector-valued ultradistribution spaces $\mathcal{D}_{L^{p}}^{\prime(s)}(U ; E)$ and $\mathcal{D}_{L^{p}, h}^{\prime(s)}(U ; E)$, where $U$ is a bounded open subset of $\mathbb{R}^{d}$. The boundedness of $U$ is important since it implies nuclearity 
of $\mathcal{D}_{L^{p}}^{(s)}(U)$ and $\mathcal{D}_{L^{p}}^{(s)}(U)$, which in turn will imply a very important kernel theorem when $E$ is equal to $\mathcal{D}_{L^{p}}^{(s)}(U)$. In the end of this section we are particularly interested in the spaces $\mathcal{D}_{L^{p}}^{(s)}(U ; E)$ when $E$ is a Banach space. All the spaces that are defined and considered in the first three sections are for the needs of the second part of the paper where we investigate the existence of various type of solutions of the Cauchy problem in spaces of Banach-valued Beurling weighted ultradistributions.

We start Section 4 by defining the Banach space $\widetilde{\mathcal{D}}_{L^{p}, h}^{\prime s}(0, T ; E)$ consisting of the sequences of Bochner $L^{p}$ functions with certain growth conditions. In this abstract setting, we define the Cauchy problem $(0.1)$ and recall from [6] two types of solutions of (0.1). Then, using the proof in [6], we prove the existence of such solutions in $\widetilde{\mathcal{D}}_{L^{p}, h}^{\prime s}(0, T ; E)$ and use this to prove existence of the solution of (0.1) in the space of Banach-valued ultradistributions $\mathcal{D}_{L^{p}}^{(s)}(0, T ; E)$.

We apply in Section 5 results of Section 4 for several important instances of $A$ and $E$ considered by Da Prato and Sinestrari in [6], but in our ultradistributional setting. The main part is the proof of the theorem that we announced above by using the theory developed in Sections 1-3.

0.1. Preliminaries. The sets of natural, integer, positive integer, real and complex numbers are denoted by $\mathbb{N}, \mathbb{Z}, \mathbb{Z}_{+}, \mathbb{R}, \mathbb{C}$. We use the symbols for $x \in \mathbb{R}^{d}:\langle x\rangle=\left(1+|x|^{2}\right)^{1 / 2}, D^{\alpha}=D_{1}^{\alpha_{1}} \cdots D_{d}^{\alpha_{d}}$, $D_{j}^{\alpha_{j}}=i^{-1} \partial^{\alpha_{j}} / \partial x^{\alpha_{j}}, \alpha=\left(\alpha_{1}, \alpha_{2}, \ldots, \alpha_{d}\right) \in \mathbb{N}^{d}$.

Let $s>1$ and $U \subseteq \mathbb{R}^{d}$ be an open set. Following Komatsu [9], for a compact set $K \subseteq \bar{U}$, define $\mathcal{E}^{s, h}(K)$ as the Banach space (from now on, abbreviated as $(B)$-space) of all $\varphi \in \mathcal{C}^{\infty}(U)$ which satisfy

$$
\sup _{\alpha \in \mathbb{N}^{d}} \sup _{x \in K} \frac{\left|D^{\alpha} \varphi(x)\right|}{h^{|\alpha|} \alpha !^{s}}<\infty
$$

and $\mathcal{D}_{K}^{s, h}$ as the $(B)$-space of all $\varphi \in \mathcal{C}^{\infty}\left(\mathbb{R}^{d}\right)$ with support in $K$, which satisfy

$$
\sup _{\alpha \in \mathbb{N}^{d}} \sup _{x \in K} \frac{\left|D^{\alpha} \varphi(x)\right|}{h^{\alpha} \alpha !^{s}}<\infty .
$$


Define the spaces

$$
\begin{aligned}
& \mathcal{E}^{(s)}(U)=\lim _{K \subset \subset \subset} \lim _{h \rightarrow 0} \mathcal{E}^{s, h}(K), \quad \mathcal{E}^{\{s\}}(U)=\lim _{K \subset \subset} \lim _{\substack{\rightarrow \\
\rightarrow \infty}} \mathcal{E}^{s, h}(K), \\
& \mathcal{D}_{K}^{(s)}=\lim _{h \rightarrow 0} \mathcal{D}_{K}^{s, h}, \quad \mathcal{D}^{(s)}(U)=\lim _{K \subset \subset \cup U} \mathcal{D}_{K}^{(s)},
\end{aligned}
$$

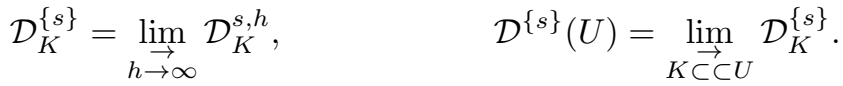

The spaces of ultradistributions and ultradistributions with compact support of Beurling and Roumieu type are defined as the strong duals of $\mathcal{D}^{(s)}(U)$ and $\mathcal{E}^{(s)}(U)$, respectively, $\mathcal{D}^{\{s\}}(U)$ and $\mathcal{E}^{\{s\}}(U)$. For the properties of these spaces we refer to $[\mathbf{9}, \mathbf{1 0}, \mathbf{1 1}]$.

It is said that

$$
P(\xi)=\sum_{\alpha \in \mathbb{N}^{d}} c_{\alpha} \xi^{\alpha}, \quad \xi \in \mathbb{R}^{d}
$$

is an ultrapolynomial of the class $(s)$, respectively $\{s\}$, whenever the coefficients $c_{\alpha}$ satisfy the estimate $\left|c_{\alpha}\right| \leq C L^{|\alpha|} / \alpha !^{s}, \alpha \in \mathbb{N}^{d}$ for some $L>0$ and $C>0$, respectively, for every $L>0$ and some $C_{L}>0$. The corresponding operator $P(D)=\sum_{\alpha} c_{\alpha} D^{\alpha}$ is an ultradifferential operator of the class $(s)$, respectively $\{s\}$, and they act continuously on $\mathcal{E}^{(s)}(U)$ and $\mathcal{D}^{(s)}(U)$, respectively $\mathcal{E}^{\{s\}}(U)$ and $\mathcal{D}^{\{s\}}(U)$, and the corresponding spaces of ultradistributions.

\section{Banach spaces of weighted ultradistributions.}

1.1. Basic Banach spaces. Let $U$ be an open subset of $\mathbb{R}^{d}$ and $1 \leq p \leq \infty$. Let $\mathcal{D}_{L^{p}, h}^{s}(U)$ be the space of all $\varphi \in \mathcal{C}^{\infty}(U)$ such that the norm

$$
\left(\sum_{\alpha \in \mathbb{N}^{d}} \frac{h^{p|\alpha|}\left\|D^{\alpha} \varphi\right\|_{L^{p}(U)}^{p}}{\alpha !^{p s}}\right)^{1 / p}
$$

is finite (with the obvious meaning when $p=\infty$ ). One can simply prove:

Lemma 1.1. $\mathcal{D}_{L^{p}, h}^{s}(U)$ is a $(B)$-space, when $1 \leq p \leq \infty$. 
Let $1 \leq p \leq \infty$ and $1 \leq q \leq \infty$ be such that

$$
\frac{1}{p}+\frac{1}{q}=1
$$

Let $\mathcal{D}_{L^{p}, h}^{(s)}(U)$ denote the closure of $\mathcal{D}^{(s)}(U)$ in $\mathcal{D}_{L^{p}, h}^{s}(U)$. Denote by $\mathcal{D}_{L^{p}, h}^{(s)}(U)$ the strong dual of $\mathcal{D}_{L^{q}, h}^{(s)}(U)$. Then, $\mathcal{D}_{L^{p}, h}^{(s)}(U)$ is continuously injected in $\mathcal{D}^{\prime(s)}(U)$, for $1 \leq p \leq \infty$. We will denote by $\mathcal{C}_{0}(U)$ the space of all continuous functions $f$ on $U$ such that, for every $\varepsilon>0$, there exists $K \subset \subset U$ such that $|f(x)|<\varepsilon$ when $x \in U \backslash K$. We leave the proof of the next lemma to the reader.

Lemma 1.2. Let $\varphi \in \mathcal{D}_{L^{\infty}, h}^{(s)}(U)$. Then, for every $\varepsilon>0$, there exist $K \subset \subset U$ and $k \in \mathbb{Z}_{+}$such that

$$
\sup _{\alpha \in \mathbb{N}^{d}} \sup _{x \in U \backslash K} \frac{h^{|\alpha|}\left|D^{\alpha} \varphi(x)\right|}{\alpha !^{s}} \leq \varepsilon \quad \text { and } \quad \sup _{|\alpha| \geq k} \frac{h^{|\alpha|}\left\|D^{\alpha} \varphi\right\|_{L^{\infty}(U)}}{\alpha !^{s}} \leq \varepsilon .
$$

1.2. Duals of Banach spaces. The main goal in this subsection is to give a representation of the elements of $\mathcal{D}_{L^{p}, h}^{(s)}(U), 1 \leq p \leq \infty$. In order to do that, first we will construct a $(B)$-space which will contain $\mathcal{D}_{L^{p}, h}^{(s)}(U)$ as a closed subspace. It is worth to note that the main idea of this construction is due to Komatsu [9].

For $1 \leq p<\infty$, define

$$
\begin{aligned}
Y_{h, L^{p}} & =\left\{\left(\psi_{\alpha}\right)_{\alpha \in \mathbb{N}^{d}} \mid \psi_{\alpha} \in L^{p}(U),\left\|\left(\psi_{\alpha}\right)_{\alpha}\right\|_{Y_{h, L^{p}}}\right. \\
& \left.=\left(\sum_{\alpha \in \mathbb{N}^{d}} \frac{h^{p|\alpha|}\left\|\psi_{\alpha}\right\|_{L^{p}(U)}^{p}}{\alpha !^{p s}}\right)^{1 / p}<\infty\right\} .
\end{aligned}
$$

Then one easily verifies that $Y_{h, L^{p}}$ is a $(B)$-space, with the norm $\|\cdot\|_{Y_{h, L} p}$, for $1 \leq p<\infty$. Let $p=\infty$. Define

$$
Y_{h, L^{\infty}}=\left\{\left(\psi_{\alpha}\right)_{\alpha \in \mathbb{N}^{d}} \mid \psi_{\alpha} \in \mathcal{C}_{0}(U), \lim _{|\alpha| \rightarrow \infty} \frac{h^{|\alpha|}\left\|\psi_{\alpha}\right\|_{L^{\infty}(U)}}{\alpha !^{s}}=0\right\},
$$

with the norm

$$
\left\|\left(\psi_{\alpha}\right)_{\alpha}\right\|_{Y_{h, L} \infty}=\sup _{\alpha \in \mathbb{N}^{d}} \frac{h^{|\alpha|}}{\alpha !^{s}}\left\|\psi_{\alpha}\right\|_{L^{\infty}(U)}
$$


One easily verifies that it is a $(B)$-space.

Let $\widetilde{U}$ be the disjoint union of countable number of copies of $U$, one for each $\alpha \in \mathbb{N}^{d}$, i.e.,

$$
\widetilde{U}=\bigsqcup_{\alpha \in \mathbb{N}^{d}} U_{\alpha}
$$

where $U_{\alpha}=U$. Equip $\widetilde{U}$ with the disjoint union topology. Then $\widetilde{U}$ is a Hausdorff locally compact space. Moreover, every open set in $\widetilde{U}$ is $\sigma$-compact. For each $1 \leq p<\infty$, one can define a Borel measure $\mu_{p}$ on $\widetilde{U}$ by

$$
\mu_{p}(E)=\sum_{\alpha} \frac{h^{|\alpha| p}}{\alpha !^{! p s}}\left|E \cap U_{\alpha}\right|,
$$

for $E$ a Borel subset of $\widetilde{U}$, where $\left|E \cap U_{\alpha}\right|$ is the Lebesgue measure of $E \cap U_{\alpha}$. It is obviously locally finite, $\sigma$-finite and $\mu(K)<\infty$ for every compact subset $K$ of $\widetilde{U}$. By the properties of $\widetilde{U}$ described above, $\mu_{p}$ is regular (both inner and outer regular). We obtained that $\mu_{p}$ is a Radon measure. It follows that $Y_{h, L^{p}}$ is exactly $L^{p}\left(\widetilde{U}, \mu_{p}\right)$, for $1 \leq p<\infty$. In particular, $Y_{h, L^{p}}$ is a reflexive $(B)$-space for $1<p<\infty$.

For $p=\infty$, we will prove that $Y_{h, L^{\infty}}$ is isomorphic to $\mathcal{C}_{0}(\widetilde{U})$. For $\psi \in \mathcal{C}_{0}(\widetilde{U})$, denote by $\psi_{\alpha}$ the restriction of $\psi$ to $U_{\alpha}$. By the definition of $\widetilde{U}, K$ is a compact subset of $\widetilde{U}$ if and only if $K \cap U_{\alpha} \neq \emptyset$ for only finitely many $\alpha \in \mathbb{N}^{d}$ and for those $\alpha, K \cap U_{\alpha}$ is a compact subset of $U_{\alpha}$. Now, one easily verifies that $\psi_{\alpha} \in \mathcal{C}_{0}(U)$ and

$$
\lim _{|\alpha| \rightarrow \infty}\left\|\psi_{\alpha}\right\|_{L^{\infty}(U)}=0
$$

Moreover, if $\psi_{\alpha} \in \mathcal{C}_{0}(U), \alpha \in \mathbb{N}^{d}$, are such that

$$
\lim _{|\alpha| \rightarrow \infty}\left\|\psi_{\alpha}\right\|_{L^{\infty}(U)}=0 .
$$

Then the function $\psi$ on $\widetilde{U}$, defined by $\psi(x)=\psi_{\alpha}(x)$, when $x \in U_{\alpha}$ is an element of $\mathcal{C}_{0}(\widetilde{U})$. We obtain that

$$
\mathcal{C}_{0}(\widetilde{U})=\left\{\left(\psi_{\alpha}\right)_{\alpha \in \mathbb{N}^{d}} \mid \psi_{\alpha} \in \mathcal{C}_{0}(U), \quad \forall \alpha \in \mathbb{N}^{d}, \lim _{|\alpha| \rightarrow \infty}\left\|\psi_{\alpha}\right\|_{L^{\infty}(U)}=0\right\}
$$

Observe that the mapping

$$
\left(\psi_{\alpha}\right)_{\alpha \in \mathbb{N}^{d}} \longmapsto\left(\widetilde{\psi}_{\alpha}\right)_{\alpha \in \mathbb{N}^{d}}
$$


where

$$
\widetilde{\psi}_{\alpha}=\frac{h^{|\alpha|}}{\alpha !^{s}} \psi_{\alpha}
$$

is an isometry from $Y_{h, L^{\infty}}$ onto $\mathcal{C}_{0}(\widetilde{U})$. For the purpose of the next proposition we will denote by $\iota$ the inverse mapping of this isometry, i.e., $\iota: \mathcal{C}_{0}(\widetilde{U}) \rightarrow Y_{h, L^{\infty}}$.

Note that $\mathcal{D}_{L^{p}, h}^{(s)}(U)$ can be identified with a closed subspace of $Y_{h, L^{p}}$ by the mapping $\varphi \mapsto\left((-D)^{\alpha} \varphi\right)_{\alpha \in \mathbb{N}^{d}}$. This is obvious for $1 \leq p<\infty$ and, for $p=\infty$, it follows from Lemma 1.2. Since $Y_{h, L^{p}}$ is reflexive for $1<p<\infty$, so is $\mathcal{D}_{L^{p}, h}^{(s)}(U)$ as a closed subspace of a reflexive $(B)$-space.

Observe that spaces $L^{p}(U)$, for $1 \leq p \leq \infty$, respectively, $\left(\mathcal{C}_{0}(U)\right)^{\prime}$, are continuously injected into $\mathcal{D}_{L^{p}, h}^{(s)}(U)$, respectively, $\mathcal{D}_{L^{1}, h}^{(s)}(U)$. For $\alpha \in \mathbb{N}^{d}$ and $F \in L^{p}(U)$, respectively, $F \in\left(\mathcal{C}_{0}(U)\right)^{\prime}$, we define $D^{\alpha} F \in \mathcal{D}_{L^{p}, h}^{(s)}(U)$, respectively, $D^{\alpha} F \in \mathcal{D}_{L^{1}, h}^{\prime(s)}(U)$, by

$$
\left\langle D^{\alpha} F, \varphi\right\rangle=\int_{U} F(x)(-D)^{\alpha} \varphi(x) d x, \quad \varphi \in \mathcal{D}_{L^{q}, h}^{(s)}(U),
$$

respectively

$$
\left\langle D^{\alpha} F, \varphi\right\rangle=\int_{U}(-D)^{\alpha} \varphi(x) d F, \quad \varphi \in \mathcal{D}_{L^{\infty}, h}^{(s)}(U) .
$$

It is easy to verify that $D^{\alpha} F$ is a well-defined element of $\mathcal{D}_{L^{p}, h}^{(s)}(U)$, respectively, $\mathcal{D}_{L^{1}, h}^{(s)}(U)$, and in fact it is equal to its ultradistributional derivative when we regard $F$ as an element of $\mathcal{D}^{\prime(s)}(U)$.

Proposition 1.3. Let $1<p \leq \infty$. For every $T \in \mathcal{D}_{L^{p}, h}^{(s)}(U)$, there exist $C>0$ and $F_{\alpha} \in L^{p}(U), \alpha \in \mathbb{N}^{d}$, such that

$$
\left(\sum_{\alpha \in \mathbb{N}^{d}} \frac{\alpha !^{p s}}{h^{|\alpha| p}}\left\|F_{\alpha}\right\|_{L^{p}(U)}^{p}\right)^{1 / p} \leq C \quad \text { and } \quad T=\sum_{|\alpha|=0}^{\infty} D^{\alpha} F_{\alpha} .
$$

When $p=1$, for every $T \in \mathcal{D}_{L^{1}, h}^{(s)}(U)$, there exist $C>0$ and Radon 
measures $F_{\alpha} \in\left(\mathcal{C}_{0}(U)\right)^{\prime}, \alpha \in \mathbb{N}^{d}$, such that

$$
\sum_{\alpha \in \mathbb{N}^{d}} \frac{\alpha !^{s}}{h^{|\alpha|}}\left\|F_{\alpha}\right\|_{\left(\mathcal{C}_{0}(U)\right)^{\prime}} \leq C \quad \text { and } \quad T=\sum_{|\alpha|=0}^{\infty} D^{\alpha} F_{\alpha} .
$$

Moreover, if $B$ is a bounded subset of $\mathcal{D}_{L^{p}, h}^{(s)}(U)$, then there exists $C>0$ independent of $T \in B$ and, for each $T \in B$ there exist $F_{\alpha} \in L^{p}(U)$, $\alpha \in \mathbb{N}^{d}$, for $1<p \leq \infty$, respectively, $F_{\alpha} \in\left(\mathcal{C}_{0}(U)\right)^{\prime}, \alpha \in \mathbb{N}^{d}$, for $p=1$, such that (1.1), respectively, (1.2), holds.

If $F_{\alpha} \in L^{p}(U), \alpha \in \mathbb{N}^{d}$, for $1<p \leq \infty$, respectively, $F_{\alpha} \in\left(\mathcal{C}_{0}(U)\right)^{\prime}$, $\alpha \in \mathbb{N}^{d}$, for $p=1$, are such that

$$
\left(\sum_{\alpha \in \mathbb{N}^{d}} \frac{\alpha !^{p s}}{h^{|\alpha| p}}\left\|F_{\alpha}\right\|_{L^{p}(U)}^{p}\right)^{1 / p}<\infty
$$

for $1<p \leq \infty$, respectively,

$$
\sum_{\alpha \in \mathbb{N}^{d}} \frac{\alpha !^{s}}{h^{|\alpha|}}\left\|F_{\alpha}\right\|_{\left(\mathcal{C}_{0}(U)\right)^{\prime}}<\infty
$$

for $p=1$, then the series

$$
\sum_{|\alpha|=0}^{\infty} D^{\alpha} F_{\alpha}
$$

converges absolutely in $\mathcal{D}_{L^{p}, h}^{\prime(s)}(U)$, respectively, $\mathcal{D}_{L^{1}, h}^{(s)}(U)$.

Proof. Let $Y_{h, L^{q}}$ be as in the above discussion. Extend $T$ by the Hahn-Banach theorem to a continuous functional on $Y_{h, L^{q}}$, and denote it again by $T$, for $1 \leq q \leq \infty$.

For $q=\infty, \widetilde{T}=T \circ \iota$ is a functional on $\mathcal{C}_{0}(\widetilde{U})$. Then, for $1<p \leq \infty$, there exists $g \in L^{p}\left(\widetilde{U}, \mu_{q}\right)$ such that

$$
T\left(\left(\psi_{\alpha}\right)_{\alpha \in \mathbb{N}^{d}}\right)=\int_{\widetilde{U}}\left(\psi_{\alpha}\right)_{\alpha \in \mathbb{N}^{d}} g d \mu_{q},
$$

$\left(\psi_{\alpha}\right)_{\alpha \in \mathbb{N}^{d}} \in Y_{h, L^{q}}$. 
For $p=1$, there exists $g \in\left(\mathcal{C}_{0}(\widetilde{U})\right)^{\prime}$ such that

$$
\widetilde{T}(\psi)=\int_{\widetilde{U}} \psi d g
$$

for $\psi \in \mathcal{C}_{0}(\widetilde{U})$. Hence, for $\left(\psi_{\alpha}\right)_{\alpha \in \mathbb{N}^{d}} \in Y_{h, L^{\infty}}$, we have

$$
T\left(\left(\psi_{\alpha}\right)_{\alpha \in \mathbb{N}^{d}}\right)=\widetilde{T}\left(\left(\widetilde{\psi}_{\alpha}\right)_{\alpha \in \mathbb{N}^{d}}\right)=\int_{\tilde{U}}\left(\widetilde{\psi}_{\alpha}\right)_{\alpha \in \mathbb{N}^{d}} d g
$$

where

$$
\left(\widetilde{\psi}_{\alpha}\right)_{\alpha}=\iota^{-1}\left(\left(\psi_{\alpha}\right)_{\alpha}\right)=\left(\frac{h^{|\alpha|}}{\alpha !^{s}} \psi_{\alpha}\right)_{\alpha}
$$

Put

$$
F_{\alpha}=\frac{h^{|\alpha| q}}{\alpha !^{q s}} g_{\mid U_{\alpha}}, \quad \text { for } 1 \leq q<\infty
$$

For $q=\infty$, put

$$
F_{\alpha}=\frac{h^{|\alpha|}}{\alpha !^{! s}} g_{\mid U_{\alpha}} .
$$

Then $F_{\alpha} \in L^{p}(U)$, for $1 \leq q<\infty$, respectively, $F_{\alpha} \in\left(\mathcal{C}_{0}(U)\right)^{\prime}$ for $q=\infty$. Moreover, for $1<q<\infty$,

$$
\sum_{\alpha \in \mathbb{N}^{d}} \frac{\alpha !^{p s}}{h^{|\alpha| p}}\left\|F_{\alpha}\right\|_{L^{p}(U)}^{p}=\sum_{\alpha \in \mathbb{N}^{d}} \frac{h^{|\alpha| q}}{\alpha !^{q s}}\left\|g_{\mid U_{\alpha}}\right\|_{L^{p}(U)}^{p}=\|g\|_{L^{p}\left(\tilde{U}, \mu_{q}\right)}^{p}<\infty .
$$

Also, it is easy to verify that, for $q=1$,

$$
\sup _{\alpha \in \mathbb{N}^{d}} \frac{\alpha !^{s}}{h^{|\alpha|}}\left\|F_{\alpha}\right\|_{L^{\infty}(U)}=\|g\|_{L^{\infty}\left(\widetilde{U}, \mu_{1}\right)}<\infty .
$$

For $q=\infty$, we have

$$
\sum_{\alpha \in \mathbb{N}^{d}} \frac{\alpha !^{s}}{h^{|\alpha|}}\left\|F_{\alpha}\right\|_{\left(\mathcal{C}_{0}(U)\right)^{\prime}}=\sum_{\alpha \in \mathbb{N}^{d}}\left\|g_{\mid U_{\alpha}}\right\|_{\left(\mathcal{C}_{0}(U)\right)^{\prime}}=\|g\|_{\left(\mathcal{C}_{0}(\widetilde{U})\right)^{\prime}}<\infty,
$$

where in the second equality we used that $\left\|g_{\mid U_{\alpha}}\right\|_{\left(\mathcal{C}_{0}(U)\right)^{\prime}}=\left|g_{\mid U_{\alpha}}\right|\left(U_{\alpha}\right)=$ $|g|\left(U_{\alpha}\right)$ (we denote by $|g|$ the total variation of the measure $g$ and similarly for $\left.g_{\mid U_{\alpha}}\right)$. Moreover,

$$
T\left((\psi)_{\alpha \in \mathbb{N}^{d}}\right)=\sum_{\alpha \in \mathbb{N}^{d}} \int_{U} \psi_{\alpha}(x) F_{\alpha}(x) d x,
$$


for $1 \leq q<\infty$. For $q=\infty$, we have

$$
\begin{aligned}
T\left(\left(\psi_{\alpha}\right)_{\alpha \in \mathbb{N}^{d}}\right) & =\int_{\tilde{U}}\left(\widetilde{\psi}_{\alpha}\right)_{\alpha \in \mathbb{N}^{d}} d g=\sum_{\alpha \in \mathbb{N}^{d}} \frac{\alpha !^{s}}{h^{|\alpha|}} \int_{U} \widetilde{\psi}_{\alpha} d F_{\alpha} \\
& =\sum_{\alpha \in \mathbb{N}^{d}} \int_{U} \psi_{\alpha} d F_{\alpha} .
\end{aligned}
$$

So, for $1 \leq q<\infty$, if $\varphi \in \mathcal{D}_{L^{q}, h}^{(s)}(U)$, we obtain

$$
\langle T, \varphi\rangle=\sum_{\alpha \in \mathbb{N}^{d}} \int_{U}(-D)^{\alpha} \varphi(x) F_{\alpha}(x) d x=\sum_{\alpha \in \mathbb{N}^{d}}\left\langle D^{\alpha} F_{\alpha}, \varphi\right\rangle .
$$

Similarly, $\langle T, \varphi\rangle=\sum_{\alpha}\left\langle D^{\alpha} F_{\alpha}, \varphi\right\rangle$ when $q=\infty$. Moreover, by these calculations, it follows that, for $1 \leq q<\infty$,

$$
\begin{aligned}
& \sum_{\alpha \in \mathbb{N}^{d}}\left|\left\langle D^{\alpha} F_{\alpha}, \varphi\right\rangle\right| \\
& \quad \leq\left(\sum_{\alpha \in \mathbb{N}^{d}} \frac{\alpha !^{p s}}{h^{|\alpha| p}}\left\|F_{\alpha}\right\|_{L^{p}(U)}^{p}\right)^{1 / p}\left(\sum_{\alpha \in \mathbb{N}^{d}} \frac{h^{|\alpha| q}\left\|D^{\alpha} \varphi\right\|_{L^{q}(U)}^{q}}{\alpha ! \varphi^{s}}\right)^{1 / q} .
\end{aligned}
$$

Hence, the partial sums of $\sum_{\alpha} D^{\alpha} F_{\alpha}$ converge absolutely in $\mathcal{D}_{L^{p}, h}^{\prime(s)}(U)$, when $1<p \leq \infty$. When $p=1$, the proof that the partial sums of $\sum_{\alpha} D^{\alpha} F_{\alpha}$ converge absolutely in $\mathcal{D}_{L^{1}, h}^{\prime(s)}(U)$ is similar and we omit it. If $B$ is a bounded subset of $\mathcal{D}_{L^{p}, h}^{(s)}(U)$, by the Hahn-Banach theorem, it can be extended to a bounded set $B_{1}$ in $Y_{h, L^{q}}^{\prime}$, for $1 \leq q<\infty$, respectively to a bounded set $B_{1}$ in $\mathcal{C}_{0}(\widetilde{U})$ for $q=\infty$ ( $\iota$ is an isometry). Hence, there exists $C>0$ independent of $T \in B_{1}$ and, for each $T \in B_{1}$, there exists $g \in L^{p}\left(\widetilde{U}, \mu_{q}\right)$, for $1<p \leq \infty$, respectively $g \in\left(\mathcal{C}_{0}(\widetilde{U})\right)^{\prime}$, for $p=1$, such that $\|g\|_{L^{p}(\widetilde{U})} \leq C$, respectively, $\|g\|_{\left(\mathcal{C}_{0}(\widetilde{U})\right)^{\prime}} \leq C$. If we define $F_{\alpha}$ as above, one obtains (1.1), respectively (1.2), with the desired uniform estimate independent of $T \in B$.

The last part of the proposition is easy, and we omit it. 


\section{Ultradistribution spaces.}

2.1. Beurling type test spaces. For $1 \leq p \leq \infty$, we define locally convex spaces (from now on abbreviated as lcs)

$$
\mathcal{B}_{L^{p}}^{(s)}(U)=\lim _{h \rightarrow \infty} \mathcal{D}_{L^{p}, h}^{s}(U) .
$$

Then $\mathcal{B}_{L^{p}}^{(s)}(U)$ is an $(F)$-space. Denote by $\mathcal{D}_{L^{p}}^{(s)}(U)$ the closure of $\mathcal{D}^{(s)}(U)$ in $\mathcal{B}_{L^{p}}^{(s)}(U)$ for $1 \leq p<\infty$ and $\dot{\mathcal{B}}^{(s)}(U)$ the closure of $\mathcal{D}^{(s)}(U)$ in $\mathcal{B}_{L^{\infty}}^{(s)}(U)$. Hence, when $U=\mathbb{R}^{d}$, these spaces coincide with the spaces

$$
\mathcal{D}_{L^{p}}^{(s)}\left(\mathbb{R}^{d}\right), \quad \text { for } 1 \leq p<\infty,
$$

respectively, $\dot{\mathcal{B}}^{(s)}$ defined in [24]. All of these spaces are $(F)$-spaces as well as

$$
X_{L^{p}}=\lim _{h \rightarrow \infty} \mathcal{D}_{L^{p}, h}^{(s)}(U), \quad 1 \leq p \leq \infty
$$

Lemma 2.1. Let $X_{L^{p}}$ be as above and $1 \leq p \leq \infty$.

(i) $\mathcal{D}^{(s)}(U)$ is dense in $X_{L^{p}}$.

(ii) $X_{L^{p}}$ is a closed subspace of $\mathcal{B}_{L^{p}}^{(s)}(U)$, and the topology of $X_{L^{p}}$ is the same as the induced one from $\mathcal{B}_{L^{p}}^{(s)}(U)$. Hence, $X_{L^{p}}$ and $\mathcal{D}_{L^{p}}^{(s)}(U)$, for $1 \leq p<\infty$, respectively, $X_{L^{\infty}}$ and $\dot{\mathcal{B}}^{(s)}(U)$ when $p=\infty$, are isomorphic lcs.

Proof. Since $\mathcal{D}^{(s)}(U)$ is dense in each $\mathcal{D}_{L^{p}, h}^{(s)}(U)$, it follows that $\mathcal{D}^{(s)}(U) \subseteq X_{L^{p}}$, and it is dense in $X_{L^{p}}$. The proof of (i) is complete.

To prove (ii), note that $X_{L^{p}} \subseteq \mathcal{B}_{L^{p}}^{(s)}(U)$. Let $\varphi_{j}, j \in \mathbb{N}$, be a sequence in $X_{L^{p}}$ which converges to $\varphi \in \mathcal{B}_{L^{p}}^{(s)}(U)$ in the topology of $\mathcal{B}_{L^{p}}^{(s)}(U)$. Then $\varphi_{j}$ converges to $\varphi$ in $\mathcal{D}_{L^{p}, h}^{s}(U)$ for each $h$. But $\varphi_{j} \in \mathcal{D}_{L^{p}, h}^{(s)}(U), j \in \mathbb{N}$ and $\mathcal{D}_{L^{p}, h}^{(s)}(U)$ is a closed subspace of $\mathcal{D}_{L^{p}, h}^{s}(U)$ with the same topology. It follows that $\varphi \in \mathcal{D}_{L^{p}, h}^{(s)}(U)$, and $\varphi_{j}$ converges to $\varphi$ in $\mathcal{D}_{L^{p}, h}^{(s)}(U)$ for each $h$. Hence, $\varphi \in X_{L^{p}}$. Moreover, since the inclusion $X_{L^{p}} \rightarrow \mathcal{B}_{L^{p}}^{(s)}(U)$ is obviously continuous and $X_{L^{p}}, \mathcal{B}_{L^{p}}^{(s)}(U)$ are $(F)$-spaces and the image of $X_{L^{p}}$ under the inclusion is a closed subspace of $\mathcal{B}_{L^{p}}^{(s)}(U)$, by the open mapping theorem, it follows that 
$X_{L^{p}}$ is isomorphic with its image under this inclusion (isomorphic as lcs).

By the above lemma, we obtain that $\mathcal{D}_{L^{p}}^{(s)}(U)=\lim _{h \rightarrow \infty} \mathcal{D}_{L^{p}, h}^{(s)}(U)$, for $1 \leq p<\infty$ and

$$
\dot{\mathcal{B}}^{(s)}(U)=\lim _{h \rightarrow \infty} \mathcal{D}_{L^{\infty}, h}^{(s)}(U), \quad \text { for } p=\infty,
$$

and the projective limits are reduced. For $1<p \leq \infty$, denote by $\mathcal{D}_{L^{p}}^{\prime(s)}(U)$ the strong dual of $\mathcal{D}_{L^{q}}^{(s)}(U)$. Denote by $\mathcal{D}_{L^{1}}^{\prime(s)}(U)$ the strong dual of $\dot{\mathcal{B}}^{(s)}(U)$. Since $\mathcal{D}^{(s)}(U)$ is continuously and densely injected into $\mathcal{D}_{L^{q}}^{(s)}(U)$, for $1 \leq q<\infty$ and into $\dot{\mathcal{B}}^{(s)}(U), \mathcal{D}_{L^{p}}^{(s)}(U)$ are continuously injected into $\mathcal{D}^{\prime(s)}(U)$, for $1 \leq p \leq \infty$. One easily verifies that ultradifferential operators of class $(s)$ act continuously on $\mathcal{D}_{L^{p}}^{(s)}(U)$, for $1 \leq p<\infty$ and on $\dot{\mathcal{B}}^{(s)}(U)$. Hence, they act continuously on $\mathcal{D}_{L^{p}}^{(s)}(U)$, for $1 \leq p \leq \infty$. For $1<p<\infty$, since all $\mathcal{D}_{L^{p}, h}^{(s)}(U)$ are reflexive $(B)$ spaces, the inclusion $\mathcal{D}_{L^{p}, h_{2}}^{(s)}(U) \rightarrow \mathcal{D}_{L^{p}, h_{1}}^{(s)}(U)$, for $h_{2}>h_{1}$, is a weakly compact mapping; hence, $\mathcal{D}_{L^{p}}^{(s)}(U)$ is an $\left(F S^{*}\right)$-space. In particular it is reflexive.

From now on, we suppose that $U$ is a bounded open set in $\mathbb{R}^{d}$.

Proposition 2.2. Let $1 \leq p<\infty$ and $h_{1}>h$. We have the continuous inclusions $\mathcal{D}_{L^{\infty}, h_{1}}^{(s)}(U) \rightarrow \mathcal{D}_{L^{p}, h}^{(s)}(U)$ and $\mathcal{D}_{L^{p}, 2^{s} h}^{(s)}(U) \rightarrow \mathcal{D}_{L^{\infty}, h}^{(s)}(U)$. In particular, the spaces $\mathcal{D}_{L^{p}}^{(s)}(U), 1 \leq p<\infty$ and $\dot{\mathcal{B}}^{(s)}(U)$ are isomorphic among each other.

Proof. Let $1 \leq p<\infty$ and $\varphi \in \mathcal{D}_{L^{p}, h}^{(s)}(U)$. It is obvious that, for each $\alpha \in \mathbb{N}^{d}, D^{\alpha} \varphi \in W_{0}^{m, p}(U)$, for any $m \in \mathbb{Z}_{+}$. Hence, by the Sobolev imbedding theorem, it follows that, for each $\alpha \in \mathbb{N}^{d}, D^{\alpha} \varphi$ extends to a uniformly continuous function on $\bar{U}$. Now, let $\varphi \in \mathcal{D}_{L^{\infty}, h_{1}}^{s}(U)$. Then

$$
\begin{aligned}
& \left(\sum_{\alpha \in \mathbb{N}^{d}} \frac{h^{p|\alpha|}\left\|D^{\alpha} \varphi\right\|_{L^{p}(U)}^{p}}{\alpha !^{p s}}\right)^{1 / p} \\
& \quad \leq|U|^{1 / p}\left(\sum_{\alpha \in \mathbb{N}^{d}} \frac{h^{p|\alpha|} h_{1}^{p|\alpha|}\left\|D^{\alpha} \varphi\right\|_{L^{\infty}(U)}^{p}}{h_{1}^{p|\alpha|} \alpha !^{p s}}\right)^{1 / p}
\end{aligned}
$$




$$
\leq C|U|^{1 / p} \sup _{\alpha \in \mathbb{N}^{d}} \frac{h_{1}^{|\alpha|}\left\|D^{\alpha} \varphi\right\|_{L^{\infty}(U)}}{\alpha !^{s}} .
$$

We obtain that the inclusion $\mathcal{D}_{L^{\infty}, h_{1}}^{s}(U) \rightarrow \mathcal{D}_{L^{p}, h}^{s}(U)$ is continuous. Moreover, if $\varphi \in \mathcal{D}_{L^{\infty}, h_{1}}^{(s)}(U)$, then there exist $\varphi_{j} \in \mathcal{D}^{(s)}(U), j \in \mathbb{Z}_{+}$, such that $\varphi_{j} \rightarrow \varphi$, as $j \rightarrow \infty$, in $\mathcal{D}_{L^{\infty}, h_{1}}^{s}(U)$. But, then, $\varphi_{j} \rightarrow \varphi$, as $j \rightarrow \infty$, in $\mathcal{D}_{L^{p}, h}^{s}(U)$. Hence, $\mathcal{D}_{L^{\infty}, h_{1}}^{(s)}(U)$ is continuously injected into $\mathcal{D}_{L^{p}, h}^{(s)}(U)$. It follows that, for each $\varphi \in \mathcal{D}_{L^{\infty}, h_{1}}^{(s)}(U), \alpha \in \mathbb{N}^{d}, D^{\alpha} \varphi$ can be extended to a uniformly continuous function on $\bar{U}$. Let $\varphi \in \mathcal{D}_{L^{p}, 2^{s} h}^{(s)}(U)$. Fix $m \in \mathbb{Z}_{+}$, such that $m p>d$. Denote by $C_{1}=\max _{|\alpha| \leq m} \alpha !^{s} / h^{|\alpha|}$. By the Sobolev imbedding theorem we have:

$$
\begin{aligned}
\frac{h^{|\beta|}\left\|D^{\beta} \varphi\right\|_{L^{\infty}(U)}}{\beta !^{s}} & \leq C^{\prime} \frac{h^{|\beta|}}{\beta !^{s}}\left(\sum_{|\alpha| \leq m}\left\|D^{\alpha+\beta} \varphi\right\|_{L^{p}(U)}^{p}\right)^{1 / p} \\
& \leq C^{\prime}\left(\sum_{|\alpha| \leq m} \frac{h^{(|\alpha|+|\beta|) p} \alpha !^{p s}}{\left.\beta !^{p s} \alpha !^{p s} h^{|\alpha| p}\left\|D^{\alpha+\beta} \varphi\right\|_{L^{p}(U)}^{p}\right)^{1 / p}}\right. \\
& \leq C^{\prime} C_{1}\left(\sum_{|\alpha| \leq m} \frac{\left(2^{s} h\right)^{(|\alpha|+|\beta|) p}}{(\alpha+\beta) ! p s}\left\|D^{\alpha+\beta} \varphi\right\|_{L^{p}(U)}^{p}\right)^{1 / p} \\
& \leq C^{\prime} C_{1}\left(\sum_{\gamma \in \mathbb{N}^{d}} \frac{\left(2^{s} h\right)^{|\gamma| p}}{\gamma !^{p s}}\left\|D^{\gamma} \varphi\right\|_{L^{p}(U)}^{p}\right)^{1 / p} .
\end{aligned}
$$

We obtain that $\mathcal{D}_{L^{p}, 2^{s} h}^{(s)}(U)$ is continuously injected in $\mathcal{D}_{L^{\infty}, h}^{s}(U)$. Moreover, if $\varphi_{j} \in \mathcal{D}^{(s)}(U), j \in \mathbb{Z}_{+}$, are such that $\varphi_{j} \rightarrow \varphi$, when $j \rightarrow \infty$, in $\mathcal{D}_{L^{p}, 2^{s} h}^{(s)}(U)$, then $\varphi_{j} \rightarrow \varphi$, when $j \rightarrow \infty$, in $\mathcal{D}_{L^{\infty}, h}^{s}(U)$. Hence, $\mathcal{D}_{L^{p}, 2^{s} h}^{(s)}(U)$ is continuously injected into $\mathcal{D}_{L^{\infty}, h}^{(s)}(U)$.

Proposition 2.2 implies that we no longer need to distinguish the spaces $\mathcal{D}_{L^{p}}^{(s)}(U)$ since they are all isomorphic to $\dot{\mathcal{B}}^{(s)}(U)$. Hence, their duals are all isomorphic to $\mathcal{D}_{L^{1}}^{(s)}(U)$.

Proposition 2.3. Let $U$ be bounded, open subset of $\mathbb{R}^{d}$.

(i) Let $h>0$ be fixed. Every element $\varphi$ of $\mathcal{D}_{L^{p}, h}^{(s)}(U)$ for $1 \leq p \leq \infty$ can be extended to a $\mathcal{C}^{\infty}$ function on $\mathbb{R}^{d}$ with support in $\bar{U}$. 
Moreover, $\mathcal{D}_{L^{\infty}, h}^{(s)}(U)$ can be identified with a closed subspace of $\mathcal{D}_{\bar{U}}^{s, h}$

(ii) $\dot{\mathcal{B}}^{(s)}(U)$ can be identified with a closed subspace of $\mathcal{D}_{\bar{U}}^{(s)}$;

(iii) $\dot{\mathcal{B}}^{(s)}(U)$ is a nuclear $(F S)$-space. Moreover, in the representation

$$
\dot{\mathcal{B}}^{(s)}(U)=\lim _{h \rightarrow \infty} \mathcal{D}_{L^{\infty}, h}^{(s)}(U),
$$

the linking inclusions in the projective limit

$$
\mathcal{D}_{L^{\infty}, h_{1}}^{(s)}(U) \rightarrow \mathcal{D}_{L^{\infty}, h}^{(s)}(U)
$$

are compact for $h_{1}>h$.

Proof. To prove the first part of (i), note that, by Proposition 2.2, $\mathcal{D}_{L^{p}, h}^{(s)}(U)$ is continuously injected into $\mathcal{D}_{L^{\infty}, h / 2^{s}}^{(s)}(U)$. Hence, it is enough to prove it for $\mathcal{D}_{L^{\infty}, h}^{(s)}(U)$. Let $\varphi \in \mathcal{D}_{L^{\infty}, h}^{(s)}(U)$. Then there exist $\varphi_{j} \in \mathcal{D}^{(s)}(U), j \in \mathbb{Z}_{+}$, such that $\varphi_{j} \rightarrow \varphi$, as $j \rightarrow \infty$ in $\mathcal{D}_{L^{\infty}, h}^{(s)}(U)$. So, for $\varepsilon>0$, there exists $j_{0} \in \mathbb{Z}_{+}$such that, for $j, k \geq j_{0}$ and $j, k \in \mathbb{Z}_{+}$, we have

$$
\sup _{\alpha \in \mathbb{N}^{d}} \frac{h^{|\alpha|}\left\|D^{\alpha} \varphi_{k}-D^{\alpha} \varphi_{j}\right\|_{L^{\infty}(U)}}{\alpha !^{s}} \leq \varepsilon .
$$

Since all $\varphi_{j}, j \in \mathbb{Z}_{+}$, have compact support in $U$ and $\mathcal{D}^{(s)}(U) \subseteq \mathcal{D}_{\bar{U}}^{s, h}$, we obtain that

$$
\sup _{\alpha \in \mathbb{N}^{d}} \frac{h^{|\alpha|}\left\|D^{\alpha} \varphi_{k}-D^{\alpha} \varphi_{j}\right\|_{L^{\infty}\left(\mathbb{R}^{d}\right)}}{\alpha !^{s}} \leq \varepsilon,
$$

for all $j, k \geq j_{0}, j, k \in \mathbb{Z}_{+}$. Hence, $\varphi_{j}$ is a Cauchy sequence in the $(B)$-space $\mathcal{D}_{\bar{U}}^{s, h}$ so it must converge to an element $\psi \in \mathcal{D}_{\bar{U}}^{s, h}$. Hence, $\psi(x)=\varphi(x)$, when $x \in U$ and obviously $\psi(x)=0$ when $x \in \mathbb{R}^{d} \backslash U$ (since all $\varphi_{j}, j \in \mathbb{Z}_{+}$, have compact support in $U$ ). This proves the first part of (i).

To prove the second part, consider the mapping $\varphi \mapsto \widetilde{\varphi}, \mathcal{D}_{L^{\infty}, h}^{(s)}(U) \rightarrow$ $\mathcal{D}_{\bar{U}}^{s, h}$, where $\widetilde{\varphi}(x)=\varphi(x)$, when $x \in U$ and $\widetilde{\varphi}(x)=0$, when $x \in \mathbb{R}^{d} \backslash U$. By the above discussion, this is a well-defined mapping. Moreover, one easily sees that it is an isometry, which completes the proof of (i). 
Observe that (ii) follows from (i) since

$$
\dot{\mathcal{B}}^{(s)}(U)=\lim _{h \rightarrow \infty} \mathcal{D}_{L^{\infty}, h}^{(s)}(U) \text { and } \mathcal{D}_{\bar{U}}^{(s)}=\lim _{h \rightarrow \infty} \mathcal{D}_{\bar{U}}^{s, h}
$$

The first part of (iii) follows from (ii) since $\dot{\mathcal{B}}^{(s)}(U)$ is a closed subspace of the nuclear $(F S)$-space $\mathcal{D}_{\bar{U}}^{(s)}$ (Komatsu in [9] proves the nuclearity of $\mathcal{D}_{\bar{U}}^{(s)}$ when $\bar{U}$ is regular compact set, but the proof is valid for general $\bar{U}$; the regularity of $\bar{U}$ is used by Komatsu [9] for the definition and nuclearity of $\mathcal{E}^{(s)}(\bar{U})$ ). For the second part, by [9, Proposition 2.2], the inclusion $\mathcal{D}_{\bar{U}}^{s, h_{1}} \rightarrow \mathcal{D}_{\bar{U}}^{s, h}$ is compact. Since $\mathcal{D}_{L^{\infty}, h_{1}}^{(s)}(U)$, respectively $\mathcal{D}_{L^{\infty}, h}^{(s)}(U)$, is a closed subspace of $\mathcal{D}_{\bar{U}}^{s, h_{1}}$, respectively $\mathcal{D}_{\bar{U}}^{s, h}$, we obtain the compactness of the inclusion under consideration.

\subsection{Weighted Beurling spaces of ultradistributions.}

Proposition 2.4. Let $T \in \mathcal{D}_{L^{1}}^{(s)}(U)$. For every $1 \leq p \leq \infty$, there exist $h, C>0$ and $F_{\alpha} \in \mathcal{C}(\bar{U}), \alpha \in \mathbb{N}^{d}$, such that

$$
\left(\sum_{\alpha \in \mathbb{N}^{d}} \frac{\alpha !^{p s}}{h^{|\alpha| p}}\left\|F_{\alpha}\right\|_{L^{\infty}(U)}^{p}\right)^{1 / p} \leq C \quad \text { and } \quad T=\sum_{\alpha \in \mathbb{N}^{d}} D^{\alpha} F_{\alpha},
$$

where the last series converges absolutely in $\mathcal{D}_{L^{1}}^{(s)}(U)$. Moreover, if $B$ is a bounded subset of $\mathcal{D}_{L^{1}}^{(s)}(U)$ and $1 \leq p \leq \infty$, then there exist $h, C>0$ independent of $T \in B$ and, for each $T \in B$, there exist $F_{\alpha} \in \mathcal{C}(\bar{U})$, $\alpha \in \mathbb{N}^{d}$, such that (2.1) holds.

Conversely, for $1 \leq p \leq \infty$, if $F_{\alpha} \in L^{p}(U), \alpha \in \mathbb{N}^{d}$, are such that

$$
\left(\sum_{\alpha \in \mathbb{N}^{d}} \frac{\alpha !^{p s}}{h^{|\alpha| p}}\left\|F_{\alpha}\right\|_{L^{p}}^{p}\right)^{1 / p}<\infty \text { for some } h>0
$$

then the series $\sum_{|\alpha|=0}^{\infty} D^{\alpha} F_{\alpha}$ converges absolutely in $\mathcal{D}_{L^{p}, h}^{(s)}(U)$, and hence also in $\mathcal{D}_{L^{1}}^{(s)}(U)$.

Proof. We will prove first the second part of the proposition. If 
$F_{\alpha} \in L^{p}(U), \alpha \in \mathbb{N}^{d}$, are as above, the absolute convergence of

$$
\sum_{|\alpha|=0}^{\infty} D^{\alpha} F_{\alpha} \quad \text { in } \mathcal{D}_{L^{p}, h}^{(s)}(U)
$$

follows by Proposition 1.3 for $1<p \leq \infty$ and can be easily verified for $p=1$. By Proposition $2.2, \dot{\mathcal{B}}^{(s)}(U)$ is continuously and densely injected into $\mathcal{D}_{L^{q}, h}^{(s)}(U)$, where $q$ is the conjugate of $p$, i.e., $p^{-1}+q^{-1}=1$ (the part about the denseness follows from the fact that $\mathcal{D}^{(s)}(U) \subseteq \dot{\mathcal{B}}^{(s)}(U)$ is dense in $\left.\mathcal{D}_{L^{q}, h}^{(s)}(U)\right)$. Hence, $\mathcal{D}_{L^{p}, h}^{(s)}(U)$ is continuously injected into $\mathcal{D}_{L^{1}}^{\prime(s)}(U)$, and we obtain that

$$
\sum_{|\alpha|=0}^{\infty} D^{\alpha} F_{\alpha}
$$

converges absolutely in $\mathcal{D}_{L^{1}}^{(s)}(U)$.

To prove the first part, we fix $1<p \leq \infty$ and let $q$ to be the conjugate of $p$. Since $\dot{\mathcal{B}}^{(s)}(U)=\lim _{h \rightarrow \infty} \mathcal{D}_{L^{\infty}, h}^{(\bar{s})}(U)$ and the projective limit is reduced with compact linking mappings (cf., Proposition 2.3),

$$
\mathcal{D}_{L^{1}}^{\prime(s)}(U)=\lim _{h \rightarrow \infty} \mathcal{D}_{L^{1}, h}^{(s)}(U) \quad \text { as lcs },
$$

where the inductive limit is injective with compact linking mappings. If $B$ is a bounded subset of $\mathcal{D}_{L^{1}}^{(s)}(U)$, there exists $h_{1}>0$ such that $B \subseteq \mathcal{D}_{L^{1}, h}^{\prime(s)}(U)$ and is bounded there. By Proposition 2.2, if we take $h=2^{s} h_{1}, \mathcal{D}_{L^{q}, h}^{(s)}(U)$ is continuously injected into $\mathcal{D}_{L^{\infty}, h_{1}}^{(s)}(U)$. Obviously, $\mathcal{D}_{L^{q}, h}^{(s)}(U)$ is dense in $\mathcal{D}_{L^{\infty}, h_{1}}^{(s)}(U)$ (since $\mathcal{D}^{(s)}(U)$ is). We obtain that $\mathcal{D}_{L^{1}, h_{1}}^{(s)}(U)$ is continuously injected into $\mathcal{D}_{L^{p}, h}^{(s)}(U)$. Hence, $B$ is a bounded subset of $\mathcal{D}_{L^{p}, h}^{(s)}(U)$. Now, by Proposition 1.3, for each $T \in B$, there exist $\widetilde{F}_{\alpha} \in L^{p}(U), \alpha \in \mathbb{N}^{d}$, such that

$$
\left(\sum_{\alpha \in \mathbb{N}^{d}} \frac{\alpha !^{p s}}{h^{p|\alpha|}}\left\|\widetilde{F}_{\alpha}\right\|_{L^{p}(U)}^{p}\right)^{1 / p} \leq C^{\prime} \quad \text { and } \quad T=\sum_{\alpha \in \mathbb{N}^{d}} D^{\alpha} \widetilde{F}_{\alpha},
$$

and the constant $C^{\prime}$ is the same for all $T \in B$. Let $L(x) \in \mathcal{C}\left(\mathbb{R}^{d}\right)$ be a 
fundamental solution of $\Delta^{d} L=\delta$ ( $\Delta$ is the Laplacian). Define

$$
G_{\alpha}(x)=\int_{U} L(x-y) \widetilde{F}_{\alpha}(y) d y, \quad \alpha \in \mathbb{N}^{d} .
$$

Obviously, $G_{\alpha} \in \mathcal{C}(\bar{U}), \alpha \in \mathbb{N}^{d}$ and $\left\|G_{\alpha}\right\|_{L^{\infty}(U)} \leq C_{1}\left\|\widetilde{F}_{\alpha}\right\|_{L^{p}(U)}$, for all $\alpha \in \mathbb{N}^{d}$. Hence,

$$
\left(\sum_{\alpha \in \mathbb{N}^{d}} \frac{\alpha !^{p s}}{h^{p|\alpha|}}\left\|G_{\alpha}\right\|_{L^{\infty}(U)}^{p}\right)^{1 / p} \leq C_{2}
$$

and $C_{2}$ is independent of $T \in B$. Let $\Delta^{d}=\sum_{\beta} c_{\beta} D^{\beta}$, and define

$$
F_{\alpha}=\sum_{\beta \leq \alpha} c_{\beta} G_{\alpha-\beta}, \quad \alpha \in \mathbb{N}^{d} .
$$

Then obviously $F_{\alpha} \in \mathcal{C}(\bar{U})$ for all $\alpha \in \mathbb{N}^{d}$. Note that $c_{\beta} \neq 0$ only for finitely many $\beta \in \mathbb{N}^{d}$. Put

$$
C_{3}=\sum_{\beta} \frac{\beta !^{s}}{h^{|\beta|}}\left|c_{\beta}\right| .
$$

Then

$$
\begin{aligned}
& \left(\sum_{\alpha \in \mathbb{N}^{d}} \frac{\alpha !^{p s}}{\left(2^{s+1} h\right)^{|\alpha| p}}\left\|F_{\alpha}\right\|_{L^{\infty}(U)}^{p}\right)^{1 / p} \\
& \leq\left(\sum_{\alpha \in \mathbb{N}^{d}} \frac{1}{2^{|\alpha| p}}\left(\sum_{\beta \leq \alpha} \frac{(\alpha-\beta) !^{s} \beta !^{s}}{h^{|\alpha|-|\beta|} h^{|\beta|}}\left|c_{\beta}\right|\left\|G_{\alpha-\beta}\right\|_{L^{\infty}(U)}\right)^{p}\right)^{1 / p} \\
& \leq C_{2} C_{3}\left(\sum_{\alpha \in \mathbb{N}^{d}} \frac{1}{2^{|\alpha| p}}\right)^{1 / p}
\end{aligned}
$$

and the last is independent of $T \in B$. Now one easily obtains that $T=\sum_{\alpha} D^{\alpha} F_{\alpha}$, which completes the first part of the proposition when $1<p \leq \infty$. Note that the case $p=1$ follows from this for any $\widetilde{h}>h$.

3. Vector-valued spaces of ultradistributions. Let now $E$ be a complete lcs. As we saw above, $\mathcal{D}_{L^{1}}^{\prime(s)}(U)$ and $\mathcal{D}_{L^{p}, h}^{(s)}(U), 1 \leq p \leq \infty$, are continuously injected into $\mathcal{D}^{\prime(s)}(U)$. Following Komatsu [11], (see 
also [16]) we define the spaces $\mathcal{D}_{L^{1}}^{(s)}(U ; E)$ and $\mathcal{D}_{L^{p}, h}^{(s)}(U ; E), 1 \leq$ $p \leq \infty$, of $E$-valued ultradistributions of type $\mathcal{D}_{L^{1}}^{(s)}(U)$ and $\mathcal{D}_{L^{p}, h}^{(s)}(U)$, respectively, as

$$
\mathcal{D}_{L^{1}}^{(s)}(U ; E)=\mathcal{D}_{L^{1}}^{\prime(s)}(U) \varepsilon E=\mathcal{L}_{\epsilon}\left(\left(\mathcal{D}_{L^{1}}^{\prime(s)}(U)\right)_{c}^{\prime}, E\right),
$$

respectively

$$
\mathcal{D}_{L^{p}, h}^{\prime(s)}(U ; E)=\mathcal{D}_{L^{p}, h}^{\prime(s)}(U) \varepsilon E=\mathcal{L}_{\epsilon}\left(\left(\mathcal{D}_{L^{p}, h}^{(s)}(U)\right)_{c}^{\prime}, E\right) .
$$

The subindex $c$ stands for the topology of compact convex circled convergence on the dual of $\mathcal{D}_{L^{1}}^{(s)}(U)$, respectively $\mathcal{D}_{L^{p}, h}^{(s)}(U)$, from the duality

$$
\left\langle\mathcal{D}_{L^{1}}^{\prime(s)}(U),\left(\mathcal{D}_{L^{1}}^{(s)}(U)\right)^{\prime}\right\rangle \text {, respectively }\left\langle\mathcal{D}_{L^{p}, h}^{(s)}(U),\left(\mathcal{D}_{L^{p}, h}^{(s)}(U)\right)^{\prime}\right\rangle .
$$

If we denote by $\iota$, respectively $\iota_{p}$, the inclusion $\mathcal{D}_{L^{1}}^{(s)}(U) \rightarrow \mathcal{D}^{\prime(s)}(U)$, respectively $\mathcal{D}_{L^{p}, h}^{(s)}(U) \rightarrow \mathcal{D}^{\prime(s)}(U)$, then $\mathcal{D}_{L^{1}}^{(s)}(U ; E)$, respectively $\mathcal{D}_{L^{p}, h}^{\prime(s)}(U ; E)$, is continuously injected into $\mathcal{D}^{\prime(s)}(U ; E)=\mathcal{D}^{\prime(s)}(U) \varepsilon E=$ $\mathcal{L}_{b}\left(\mathcal{D}^{(s)}(U), E\right)$ by the mapping $\iota \varepsilon$ Id, respectively $\iota_{p} \varepsilon \operatorname{Id}($ cf., [11]). In [29], it is proved that when both spaces are complete, the same holds for their $\varepsilon$ tensor product. Hence, $\mathcal{D}_{L^{1}}^{\prime(s)}(U ; E)$ and $\mathcal{D}_{L^{p}, h}^{(s)}(U ; E)$ are complete. Since $\mathcal{D}_{L^{1}}^{\prime(s)}(U)$ and $\mathcal{D}_{L^{p}, h}^{(s)}(U)$ are barrelled (the former is a $(D F S)$-space as the strong dual of a $(F S)$-space, hence barrelled), every bounded subset of $\left(\mathcal{D}_{L^{1}}^{\prime(s)}(U)\right)_{c}^{\prime}$ or $\left(\mathcal{D}_{L^{p}, h}^{(s)}(U)\right)_{c}^{\prime}$ is equicontinuous (and vice versa). Hence, the $\epsilon$ topology on the right hand sides of (3.1) and (3.2) is the same as the topology of bounded convergence. Moreover, since $\dot{\mathcal{B}}^{(s)}(U)$ is an $(F S)$-space and $\mathcal{D}_{L^{1}}^{(s)}(U)$ is a $(D F S)$-space, they are both Montel spaces. Hence, $\mathcal{D}_{L^{1}}^{(s)}(U ; E)=\mathcal{L}_{b}\left(\dot{\mathcal{B}}^{(s)}(U), E\right)$. For $1<$ $p<\infty, \mathcal{D}_{L^{p}, h}^{(s)}(U ; E)=\mathcal{L}_{b}\left(\mathcal{D}_{L^{q}, h}^{(s)}(U)_{c}, E\right)$, since $\mathcal{D}_{L^{q}, h}^{(s)}(U)$ are reflexive, where $\mathcal{D}_{L^{q}, h}^{(s)}(U)_{c}$ is the space $\mathcal{D}_{L^{q}, h}^{(s)}(U)$ equipped with topology of compact convex circled convergence from the duality $\left\langle\mathcal{D}_{L^{q}, h}^{(s)}(U), \mathcal{D}_{L^{p}, h}^{(s)}(U)\right\rangle$.

Since $\dot{\mathcal{B}}^{(s)}(U)$ is a nuclear $(F S)$-space (by Proposition 2.3) $\mathcal{D}_{L^{1}}^{(s)}(U)$ is a nuclear $(D F S)$-space, and hence it satisfies the weak approximation 
property by [27, Corollary 2, page 110] (for the definition of the weak approximation property see [29]). Hence, [11, Proposition 1.4] implies $\mathcal{D}_{L^{1}}^{(s)}(U ; E)=\mathcal{D}_{L^{1}}^{(s)}(U) \varepsilon E \cong \mathcal{D}_{L^{1}}^{\prime(s)}(U) \widehat{\otimes} E$ where the $\pi$ and the $\epsilon$ topologies coincide on $\mathcal{D}_{L^{1}}^{(s)}(U) \widehat{\otimes} E$ since $\mathcal{D}_{L^{1}}^{\prime(s)}(U)$ is nuclear. We stress that here the nuclearity of the space $\mathcal{D}_{L^{1}}^{\prime(s)}(U)$ is crucial for the isomorphism $\mathcal{D}_{L^{1}}^{\prime(s)}(U) \varepsilon E \cong \mathcal{D}_{L^{1}}^{\prime(s)}(U) \widehat{\otimes} E$, (cf., [11, Theorem 2.2, page $669]$ ), a fact which is extensively used in the rest of the paper. The following kernel theorem, which plays an essential role in the proof of Theorem 5.4, also relies on the nuclearity of $\mathcal{D}_{L^{1}}^{\prime(s)}(U)$.

Theorem 3.1. Let $U_{1}$ and $U_{2}$ be bounded open sets in $\mathbb{R}_{x}^{d_{1}}$ and $\mathbb{R}_{y}^{d_{2}}$, respectively. Then we have the following canonical isomorphisms of lcs:

(i) $\dot{\mathcal{B}}^{(s)}\left(U_{1}\right) \widehat{\otimes} \dot{\mathcal{B}}^{(s)}\left(U_{2}\right) \cong \dot{\mathcal{B}}^{(s)}\left(U_{1} \times U_{2}\right)$.

(ii) $\mathcal{D}_{L^{1}}^{(s)}\left(U_{1}\right) \widehat{\otimes} \mathcal{D}_{L^{1}}^{(s)}\left(U_{2}\right) \cong \mathcal{D}_{L^{1}}^{(s)}\left(U_{1} \times U_{2}\right) \cong \mathcal{D}_{L^{1}}^{\prime(s)}\left(U_{1}\right) \varepsilon \mathcal{D}_{L^{1}}^{(s)}\left(U_{2}\right) \cong$ $\mathcal{L}_{b}\left(\dot{\mathcal{B}}^{(s)}\left(U_{1}\right), \mathcal{D}_{L^{1}}^{\prime(s)}\left(U_{2}\right)\right) \cong \mathcal{D}_{L^{1}}^{(s)}\left(U_{1} ; \mathcal{D}_{L^{1}}^{(s)}\left(U_{2}\right)\right) \cong \mathcal{D}_{L^{1}}^{(s)}\left(U_{2} ; \mathcal{D}_{L^{1}}^{\prime(s)}\left(U_{1}\right)\right)$.

Proof. First we prove (i). Since $\dot{\mathcal{B}}^{(s)}\left(U_{1}\right)$ and $\dot{\mathcal{B}}^{(s)}\left(U_{2}\right)$ are nuclear (Proposition 2.3) the $\pi$ and the $\epsilon$ topologies coincide on $\dot{\mathcal{B}}^{(s)}\left(U_{1}\right) \otimes$ $\dot{\mathcal{B}}^{(s)}\left(U_{2}\right)$. Moreover, one easily verifies that $\dot{\mathcal{B}}^{(s)}\left(U_{1}\right) \otimes \dot{\mathcal{B}}^{(s)}\left(U_{2}\right)$ can be regarded as a subspace of $\dot{\mathcal{B}}^{(s)}\left(U_{1} \times U_{2}\right)$ by identifying $\varphi \otimes \psi$ with $\varphi(x) \psi(y)$. Since $\mathcal{D}^{(s)}\left(U_{1} \times U_{2}\right)$ is continuously and densely injected in $\dot{\mathcal{B}}^{(s)}\left(U_{1} \times U_{2}\right)$ and $\mathcal{D}^{(s)}\left(U_{1}\right) \otimes \mathcal{D}^{(s)}\left(U_{2}\right)$ is a dense subspace of $\mathcal{D}^{(s)}\left(U_{1} \times\right.$ $U_{2}$ ) (see [10, Theorem 2.1]) we obtain that $\mathcal{D}^{(s)}\left(U_{1}\right) \otimes \mathcal{D}^{(s)}\left(U_{2}\right)$, and hence $\dot{\mathcal{B}}^{(s)}\left(U_{1}\right) \otimes \dot{\mathcal{B}}^{(s)}\left(U_{2}\right)$ is a dense subspace of $\dot{\mathcal{B}}^{(s)}\left(U_{1} \times U_{2}\right)$. Observe that the bilinear mapping $(\varphi, \psi) \mapsto \varphi(x) \psi(y), \dot{\mathcal{B}}^{(s)}\left(U_{1}\right) \times$ $\dot{\mathcal{B}}^{(s)}\left(U_{2}\right) \rightarrow \dot{\mathcal{B}}^{(s)}\left(U_{1} \times U_{2}\right)$ is continuous (it is separately continuous, and hence continuous, since all spaces under consideration are $(F)$ spaces). We obtain that the $\pi$ topology on $\dot{\mathcal{B}}^{(s)}\left(U_{1}\right) \otimes \dot{\mathcal{B}}^{(s)}\left(U_{2}\right)$ is stronger than the induced one by $\dot{\mathcal{B}}^{(s)}\left(U_{1} \times U_{2}\right)$. Hence, to obtain $\dot{\mathcal{B}}^{(s)}\left(U_{1}\right) \widehat{\otimes} \dot{\mathcal{B}}^{(s)}\left(U_{2}\right) \cong \dot{\mathcal{B}}^{(s)}\left(U_{1} \times U_{2}\right)$, it is enough to prove that the $\epsilon$ topology on $\dot{\mathcal{B}}^{(s)}\left(U_{1}\right) \otimes \dot{\mathcal{B}}^{(s)}\left(U_{2}\right)$ is weaker than the induced one by $\dot{\mathcal{B}}^{(s)}\left(U_{1} \times U_{2}\right)$. Let $A^{\prime}$ and $B^{\prime}$ be equicontinuous subsets of $\mathcal{D}_{L^{1}}^{\prime(s)}\left(U_{1}\right)$ 
and $\mathcal{D}_{L^{1}}^{\prime(s)}\left(U_{2}\right)$, respectively. Hence, there exist $h, C>0$ such that

$$
\sup _{T \in A^{\prime}}|\langle T, \varphi\rangle| \leq C \sup _{x, \alpha} \frac{h^{|\alpha|}\left|D^{\alpha} \varphi(x)\right|}{\alpha !^{s}}
$$

and

$$
\sup _{S \in B^{\prime}}|\langle S, \psi\rangle| \leq C \sup _{y, \beta} \frac{h^{|\beta|}\left|D^{\beta} \psi(y)\right|}{\beta !^{s}} .
$$

Then, for $\chi \in \dot{\mathcal{B}}^{(s)}\left(U_{1}\right) \otimes \dot{\mathcal{B}}^{(s)}\left(U_{2}\right), T \in A^{\prime}$ and $S \in B^{\prime}$, we have

$$
\begin{aligned}
\mid\langle T(x) & \otimes S(y), \chi(x, y)\rangle|=|\langle T(x),\langle S(y), \chi(x, y)\rangle\rangle \mid \\
& \leq C \sup _{x, \alpha} \frac{h^{|\alpha|}\left|\left\langle S(y), D_{x}^{\alpha} \chi(x, y)\right\rangle\right|}{\alpha !^{s}} \\
& \leq C^{2} \sup _{x, y, \alpha, \beta} \frac{h^{|\alpha|+|\beta|}\left|D_{x}^{\alpha} D_{y}^{\beta} \chi(x, y)\right|}{\alpha !^{!} \beta !^{s}} \\
& \leq C^{2} \sup _{x, y, \alpha, \beta} \frac{\left(2^{s} h\right)^{|\alpha|+|\beta|}\left|D_{x}^{\alpha} D_{y}^{\beta} \chi(x, y)\right|}{(\alpha+\beta) !^{s}} .
\end{aligned}
$$

Hence, we obtain that the $\epsilon$ topology is weaker than the topology induced by $\dot{\mathcal{B}}^{(s)}\left(U_{1} \times U_{2}\right)$.

(ii) Since $\dot{\mathcal{B}}^{(s)}\left(U_{1}\right)$ and $\dot{\mathcal{B}}^{(s)}\left(U_{2}\right)$ are nuclear $(F S)$-spaces (by Proposition 2.3), $\mathcal{D}_{L^{1}}^{\prime(s)}\left(U_{1}\right)$ and $\mathcal{D}_{L^{1}}^{\prime(s)}\left(U_{2}\right)$ are nuclear $(D F S)$-spaces. Hence, the $\pi$ and the $\epsilon$ topologies on the tensor product $\mathcal{D}_{L^{1}}^{\prime(s)}\left(U_{1}\right) \otimes \mathcal{D}_{L^{1}}^{\prime(s)}\left(U_{2}\right)$ coincide and, by (i) (using the fact that $\mathcal{D}_{L^{1}}^{(s)}\left(U_{1}\right)$ and $\mathcal{D}_{L^{1}}^{\prime(s)}\left(U_{2}\right)$ are nuclear ( $D F S)$-spaces), we have

$$
\mathcal{D}_{L^{1}}^{\prime(s)}\left(U_{1} \times U_{2}\right) \cong\left(\dot{\mathcal{B}}^{(s)}\left(U_{1}\right) \widehat{\otimes} \dot{\mathcal{B}}^{(s)}\left(U_{2}\right)\right)^{\prime} \cong \mathcal{D}_{L^{1}}^{(s)}\left(U_{1}\right) \widehat{\otimes} \mathcal{D}_{L^{1}}^{\prime(s)}\left(U_{2}\right) .
$$

Other isomorphisms in the assertion on $U$ follow by the discussion before the theorem.

3.1. Banach-valued ultradistributions. Now let $E$ be a $(B)$-space and denote by $L^{p}(U ; E), 1 \leq p \leq \infty$, the Bochner $L^{p}$ space. If $\varphi \in \mathcal{C}_{L^{\infty}}(U)$ (the space of bounded continuous functions on $U$ ) and $\mathbf{F} \in L^{1}(U ; E)$, then one easily verifies that $\varphi \mathbf{F} \in L^{1}(U ; E)$. We will need the following lemma. 
Lemma 3.2. (variant of du Bois-Reymond lemma for Bochner integrable functions). Let $\mathbf{F} \in L^{1}(U ; E)$ be such that

$$
\int_{U} \mathbf{F}(x) \varphi(x) d x=0
$$

for all $\varphi \in \mathcal{D}^{(s)}(U)$. Then $\mathbf{F}(x)=0$ almost everywhere.

Proof. Observe first that, for each $e^{\prime} \in E^{\prime}$ and $\varphi \in \mathcal{D}^{(s)}(U)$, we have

$$
\int_{U} e^{\prime} \circ \mathbf{F}(x) \varphi(x) d x=e^{\prime}\left(\int_{U} \mathbf{F}(x) \varphi(x) d x\right)=0 .
$$

Since $\mathcal{D}^{(s)}(U)$ is dense in $\mathcal{D}(U)$, by the du Bois-Reymond lemma, it follows that $e^{\prime} \circ \mathbf{F}=0$ almost everywhere for each $e^{\prime} \in E^{\prime}$. Since $\mathbf{F}$ is strongly measurable, $\mathbf{F}(U)$ is a separable subset of $E$.

Let $D$ be a countable dense subset of $\mathbf{F}(U)$. Denote by $L$ the set of all finite linear combinations of the elements of $D$ with scalars from $\mathbb{Q}+i \mathbb{Q}$. Then $L$ is countable. Denote by $\widetilde{E}$ the closure of $L$ in $E$. Then $\widetilde{E}$ is a separable $(B)$-space and $\mathbf{F}(U) \subseteq \widetilde{E}$. Thus, $\widetilde{E}_{\sigma}^{\prime}$ is separable (by [27, Theorem 1.7, Chapter 4]; $\sigma$ stands for the weak* topology). Let $\widetilde{V}=\left\{\widetilde{e}_{1}^{\prime}, \widetilde{e}_{2}^{\prime}, \widetilde{e}_{3}^{\prime}, \ldots\right\}$ be a countable dense subset of $\widetilde{E}_{\sigma}^{\prime}$. Extend each $\widetilde{e}_{j}^{\prime}, j \in \mathbb{Z}_{+}$, by the Hahn-Banach theorem to a continuous functional of $E$, and denote this extension by $e_{j}^{\prime}, j \in \mathbb{Z}_{+}$. Arguments given above imply that $e_{j}^{\prime} \circ \mathbf{F}=0$ almost everywhere for each $j \in \mathbb{Z}_{+}$and, in fact, $\widetilde{e}_{j}^{\prime} \circ \mathbf{F}=0$ almost everywhere, $j \in \mathbb{Z}_{+}$, since $e_{j}^{\prime}$ is extension of $\widetilde{e}_{j}^{\prime}$ and $\mathbf{F}(U) \subseteq \widetilde{E}$. Hence, $P_{j}=\left\{x \in U \mid \widetilde{e}_{j}^{\prime} \circ \mathbf{F}(x) \neq 0\right\}$ is a set of measure 0 for each $j \in \mathbb{Z}_{+}$and so is $P=\bigcup_{j} P_{j}$.

We will prove that $\mathbf{F}(x)=0$ for every $x \in U \backslash P$. Assume that there exists $x_{0} \in U \backslash P$ such that $\mathbf{F}\left(x_{0}\right) \neq 0$. Then there exists $\widetilde{e}^{\prime} \in \widetilde{E}^{\prime}$ such that $\widetilde{e}^{\prime} \circ \mathbf{F}\left(x_{0}\right) \neq 0$, i.e., $\left|\widetilde{e}^{\prime} \circ \mathbf{F}\left(x_{0}\right)\right|=c>0$. Then there exists $\widetilde{e}_{k}^{\prime} \in \widetilde{V}$ such that $\left|\widetilde{e}^{\prime} \circ \mathbf{F}\left(x_{0}\right)-\widetilde{e}_{k} \circ \mathbf{F}\left(x_{0}\right)\right| \leq c / 2$. Since $\widetilde{e}_{k}^{\prime} \circ \mathbf{F}\left(x_{0}\right)=0$, by the definition of $P$, we have

$$
c=\left|\widetilde{e}^{\prime} \circ \mathbf{F}\left(x_{0}\right)\right| \leq\left|\widetilde{e}^{\prime} \circ \mathbf{F}\left(x_{0}\right)-\widetilde{e}_{k}^{\prime} \circ \mathbf{F}\left(x_{0}\right)\right|+\left|\widetilde{e}_{k}^{\prime} \circ \mathbf{F}\left(x_{0}\right)\right| \leq c / 2,
$$

which is a contradiction. Hence, $\mathbf{F}(x)=0$ for all $x \in U \backslash P$, and the proof is complete. 
Denote by $\delta_{x}$ the delta ultradistribution concentrated at $x$. For $\alpha \in \mathbb{N}^{d}$ and $x \in U$ one easily verifies that $D^{\alpha} \delta_{x} \in \mathcal{D}_{L^{1}, h}^{(s)}(U)$ for any $h>0$ and, hence, by Proposition $2.2, D^{\alpha} \delta_{x} \in \mathcal{D}_{L^{p}, h}^{(s)}(U)$ for any $h>0$ and $1 \leq p \leq \infty$. For the next proposition, we need the following result.

Lemma 3.3. Let $h>0, \alpha \in \mathbb{N}^{d}$ and $1 \leq p \leq \infty$. The set $G_{\alpha}=\left\{D^{\alpha} \delta_{x} \mid x \in U\right\} \subseteq \mathcal{D}_{L^{p}, h}^{(s)}(U)$ is precompact in $\mathcal{D}_{L^{p}, h}^{(s)}(U)$.

Proof. Let $0<h_{1}<h / 2^{s}$. By Proposition 2.2, we have the continuous inclusion $\mathcal{D}_{L^{q}, h}^{(s)}(U) \rightarrow \mathcal{D}_{L^{\infty}, h / 2^{s}}^{(s)}(U)$. Proposition 2.3 implies that the inclusion $\mathcal{D}_{L^{\infty}, h / 2^{s}}^{(s)}(U) \rightarrow \mathcal{D}_{L^{\infty}, h_{1}}^{(s)}(U)$ is compact. Hence, we have the compact dense inclusion $\mathcal{D}_{L^{q}, h}^{(s)}(U) \rightarrow \mathcal{D}_{L^{\infty}, h_{1}}^{(s)}(U)$ (the denseness follows from the fact that $\mathcal{D}^{(s)}(U) \subseteq \mathcal{D}_{L^{q}, h}^{(s)}(U)$ is dense in $\left.\mathcal{D}_{L^{\infty}, h_{1}}^{(s)}(U)\right)$. So, the dual mapping $\mathcal{D}_{L^{1}, h_{1}}^{\prime(s)}(U) \rightarrow \mathcal{D}_{L^{p}, h}^{(s)}(U)$ is a compact inclusion. Observe that, for $\varphi \in \mathcal{D}_{L^{\infty}, h_{1}}^{(s)}(U)$,

$$
\left|\left\langle D^{\alpha} \delta_{x}, \varphi\right\rangle\right| \leq \frac{\alpha !^{s}}{h_{1}^{|\alpha|}}\left\|D^{\alpha} \varphi\right\|_{\mathcal{D}_{L, h_{1}}^{(s)}(U)},
$$

for all $x \in U$. Hence, $G_{\alpha}$ is bounded in the $(B)$-space $\mathcal{D}_{L^{1}, h_{1}}^{(s)}(U)$, thus precompact in $\mathcal{D}_{L^{p}, h}^{(s)}(U)$.

Proposition 3.4. Each $\mathbf{F} \in L^{p}(U ; E)$ can be regarded as an E-valued ultradistribution by

$$
\overline{\mathbf{F}}(\varphi)=\int_{U} \mathbf{F}(x) \varphi(x) d x
$$

In this way, $L^{p}(U ; E)$ is continuously injected into $\mathcal{D}_{L^{1}}^{(s)}(U ; E)$ for $1 \leq p \leq \infty$ and in $\mathcal{D}_{L^{p}, h}^{(s)}(U ; E)$ for $1<p<\infty$.

Proof. Let $\mathbf{F} \in L^{p}(U ; E)$. First, we will prove that $L^{p}(U ; E)$ is 
continuously injected into $\mathcal{D}_{L^{1}}^{\prime(s)}(U ; E)$. If $\varphi \in \dot{\mathcal{B}}^{(s)}(U)$, then

$$
\begin{aligned}
\left\|\int_{U} \mathbf{F}(x) \varphi(x) d x\right\|_{E} & \leq \int_{U}\|\mathbf{F}(x)\|_{E}|\varphi(x)| d x \\
& \leq\|\mathbf{F}\|_{L^{p}(U ; E)}\|\varphi\|_{L^{q}(U)} .
\end{aligned}
$$

Since $U$ is bounded, $\|\varphi\|_{L^{q}(U)} \leq|U|^{1 / q}\|\varphi\|_{L^{\infty}(U)}$. Hence,

$$
\overline{\mathbf{F}} \in \mathcal{L}_{b}\left(\dot{\mathcal{B}}^{(s)}(U), E\right)=\mathcal{D}_{L^{1}}^{(s)}(U ; E)
$$

and the mapping $\mathbf{F} \mapsto \overline{\mathbf{F}}$ is continuous from $L^{p}(U ; E)$ into $\mathcal{D}_{L^{1}}^{\prime(s)}(U ; E)$. To prove that it is injective let $\overline{\mathbf{F}}=0$, i.e.,

$$
\int_{U} \mathbf{F}(x) \varphi(x) d x=0
$$

for all $\varphi \in \dot{\mathcal{B}}^{(s)}(U)$. Since $U$ is bounded, $L^{p}(U ; E) \subseteq L^{1}(U ; E)$. Now, Lemma 3.2 implies that $\mathbf{F}=0$.

Next, we prove that $L^{p}(U ; E)$ is continuously injected into $\mathcal{D}_{L^{p}, h}^{(s)}(U ; E)$ for $1<p<\infty$. Consider the set $G=\left\{\delta_{x} \mid x \in U\right\} \subseteq \mathcal{D}_{L^{p}, h}^{(s)}(U)$. It is precompact in $\mathcal{D}_{L^{p}, h}^{(s)}(U)$ by Lemma 3.3. Fix $\mathbf{F} \in L^{p}(U ; E)$, and note that (3.3) still holds when $\varphi \in \mathcal{D}_{L^{q}, h}^{(s)}(U)$. Let $V=\left\{e \in E \mid\|e\|_{E} \leq \varepsilon\right\}$ be a neighborhood of zero in $E$ and

$$
\widetilde{G}=\frac{\|\mathbf{F}\|_{L^{p}(U ; E)}|U|^{1 / q}}{\varepsilon} G .
$$

Since $G$ is precompact, so is $\widetilde{G}$. But then, for $\varphi \in \widetilde{G}^{\circ}$,

$$
\|\mathbf{F}\|_{L^{p}(U ; E)}\|\varphi\|_{L^{q}(U)} \leq|U|^{1 / q}\|\mathbf{F}\|_{L^{p}(U ; E)} \sup _{x \in U}\left|\left\langle\delta_{x}, \varphi\right\rangle\right| \leq \varepsilon .
$$

Hence, $\overline{\mathbf{F}}(\varphi) \in V$ for all $\varphi \in \widetilde{G}^{\circ}$. We obtain that $\overline{\mathbf{F}} \in \mathcal{L}\left(\mathcal{D}_{L^{q}, h}^{(s)}(U)_{c}, E\right)$ since the topology of precompact convergence on $\mathcal{D}_{L^{q}, h}^{(s)}(U)$ coincides with the topology of compact convex circled convergence $\left(\mathcal{D}_{L^{p}, h}^{(s)}(U)\right.$ is a (B)-space). The continuity of the mapping $\mathbf{F} \mapsto \overline{\mathbf{F}}$ follows from (3.3) since the bounded sets of $\mathcal{D}_{L^{q}, h}^{(s)}(U)$ are the same for the initial topology and the topology of compact convex circled convergence. The proof of the injectivity is the same as above. 
By Proposition 3.4, from now on we will use the same notation for $\mathbf{F} \in L^{p}(U ; E)$ and its image in $\mathcal{D}_{L^{1}}^{(s)}(U ; E)$, respectively $\mathcal{D}_{L^{p}, h}^{(s)}(U ; E)$ for $1<p<\infty$.

For $\alpha \in \mathbb{N}^{d}$ and $\mathbf{F} \in L^{p}(U ; E), 1<p<\infty$, define $D^{\alpha} \mathbf{F} \in$ $\mathcal{D}_{L^{p}, h}^{(s)}(U ; E)$ by

$$
D^{\alpha} \mathbf{F}(\varphi)=\int_{U} \mathbf{F}(x)(-D)^{\alpha} \varphi(x) d x, \quad \varphi \in \mathcal{D}_{L^{q}, h}^{(s)}(U) .
$$

As in Proposition 3.4, one can prove that this is a well-defined element of $\mathcal{D}_{L^{p}, h}^{(s)}(U ; E)$. One only has to use the set $G_{\alpha}$ from Lemma 3.3 instead of $G=\left\{\delta_{x} \mid x \in U\right\}$. Observe that $D^{\alpha} \mathbf{F}$ coincides with the ultradistributional derivative of $\mathbf{F}$ when we regard $\mathbf{F}$ as an element of $\mathcal{D}_{L^{1}}^{(s)}(U ; E)$ or $\mathcal{D}^{\prime(s)}(U ; E)$.

Theorem 3.5. Let $1<p<\infty$ and $\mathbf{F}_{\alpha} \in L^{p}(U ; E), \alpha \in \mathbb{N}^{d}$, be such that, for some fixed $h>0$,

$$
\left(\sum_{\alpha} \frac{\alpha !^{p s}}{h^{|\alpha| p}}\left\|\mathbf{F}_{\alpha}\right\|_{L^{p}}^{p}\right)^{1 / p}<\infty .
$$

Then the partial sums $\sum_{|\alpha|=0}^{n} D^{\alpha} \mathbf{F}_{\alpha}$ converge absolutely in $\mathcal{D}_{L^{p}}^{(s)}(U ; E)$ and $\mathcal{D}_{L^{p}, h}^{\prime(s)}(U ; E)$.

The partial sums converge absolutely in $\mathcal{D}_{L^{1}}^{\prime(s)}(U ; E)$ also in the cases $p=1$ and $p=\infty$.

Proof. Let $1<p<\infty$. To prove that the partial sums converge absolutely in $\mathcal{D}_{L^{p}, h}^{(s)}(U ; E)=\mathcal{L}_{b}\left(\mathcal{D}_{L^{q}, h}^{(s)}(U)_{c}, E\right)$, let $B$ be a bounded subset of $\mathcal{D}_{L^{q}, h}^{(s)}(U)_{c}$. Since the bounded sets of $\mathcal{D}_{L^{q}, h}^{(s)}(U)$ are the same for the initial topology and the topology of compact convex circled convergence we may assume that $B$ is the closed unit ball in $\mathcal{D}_{L^{q}, h}^{(s)}(U)$. We obtain:

$$
\begin{aligned}
\sum_{|\alpha|=0}^{n} \sup _{\varphi \in B}\left\|\int_{U} \mathbf{F}_{\alpha}(x)(-D)^{\alpha} \varphi(x) d x\right\|_{E} \\
\leq \sup _{\varphi \in B} \sum_{|\alpha|=0}^{\infty} \int_{U}\left\|\mathbf{F}_{\alpha}(x)\right\|_{E}\left|D^{\alpha} \varphi(x)\right| d x
\end{aligned}
$$




$$
\begin{aligned}
& \leq \sup _{\varphi \in B} \sum_{|\alpha|=0}^{\infty}\left\|\mathbf{F}_{\alpha}\right\|_{L^{p}(U ; E)}\left\|D^{\alpha} \varphi\right\|_{L^{q}(U)} \\
& \leq\left(\sum_{|\alpha|=0}^{\infty} \frac{\alpha !^{p s}}{h^{|\alpha| p}}\left\|\mathbf{F}_{\alpha}\right\|_{L^{p}(U ; E)}^{p}\right)^{1 / p} \\
& \cdot \sup _{\varphi \in B}\left(\sum_{|\alpha|=0}^{\infty} \frac{h^{|\alpha| q}}{\alpha !^{q s}}\left\|D^{\alpha} \varphi\right\|_{L^{q}(U)}^{q}\right)^{1 / q}
\end{aligned}
$$

for any $n \in \mathbb{Z}_{+}$. Since $\mathcal{D}_{L^{p}, h}^{(s)}(U ; E)$ is complete, it follows that the partial sums converge absolutely in $\mathcal{D}_{L^{p}, h}^{(s)}(U ; E)$ to an element of $\mathcal{D}_{L^{p}, h}^{\prime(s)}(U ; E)$. The proof for $\mathcal{D}_{L^{1}}^{\prime(s)}(U ; E)$ is similar.

Observe that each $\mathbf{F} \in \mathcal{C}(\bar{U} ; E)$ is in $L^{p}(U ; E)$ for any $1 \leq p \leq \infty$. To see this, note that $\mathbf{F}$ is separately valued since it is continuous and $\bar{U}$ is a subset of $\mathbb{R}^{d}$. Moreover, it is easy to see that it is weakly measurable. Hence, Pettis's theorem implies that $\mathbf{F}$ is strongly measurable. Now the claim follows since $U$ is bounded $\|\mathbf{F}(\cdot)\|_{E}$ is in $L^{p}(U)$, for any $1 \leq p \leq \infty$.

Theorem 3.6. Let $\mathbf{f} \in \mathcal{D}_{L^{1}}^{(s)}(U ; E)$ and $1 \leq p \leq \infty$. Then there exists $h>0$ and $\mathbf{F}_{\alpha} \in \mathcal{C}(\bar{U} ; E), \alpha \in \mathbb{N}^{d}$, such that

$$
\left(\sum_{\alpha} \frac{\alpha !^{p s}}{h^{|\alpha| p}}\left\|\mathbf{F}_{\alpha}\right\|_{L^{p}(U ; E)}^{p}\right)^{1 / p}<\infty
$$

and

$$
\mathbf{f}=\sum_{|\alpha|=0}^{\infty} D^{\alpha} \mathbf{F}_{\alpha}
$$

where the series converges absolutely in $\mathcal{D}_{L^{1}}^{(s)}(U ; E)$.

Conversely, let $\mathbf{F}_{\alpha} \in L^{p}(U ; E), \alpha \in \mathbb{N}^{d}$, be such that (3.4) holds. Then there exists $\mathbf{f} \in \mathcal{D}_{L^{1}}^{(s)}(U ; E)$ such that $\mathbf{f}=\sum_{|\alpha|=0}^{\infty} D^{\alpha} \mathbf{F}_{\alpha}$ and the series converges absolutely in $\mathcal{D}_{L^{1}}^{\prime(s)}(U ; E)$.

Proof. First, note that the second part of the theorem follows by Theorem 3.5. 
To prove the first part, let $\mathbf{f} \in \mathcal{D}_{L^{1}}^{\prime(s)}(U ; E)=\mathcal{L}_{b}\left(\dot{\mathcal{B}}^{(s)}(U), E\right)$. Since $\dot{\mathcal{B}}^{(s)}(U)$ is nuclear (by Proposition 2.3) and $E$ is a $(B)$-space, $\mathbf{f}$ is nuclear. Hence, there exists a sequence $e_{j}, j \in \mathbb{N}$, in the closed unit ball of $E$, an equicontinuous sequence $f_{j}, j \in \mathbb{N}$, of $\mathcal{D}_{L^{1}}^{(s)}(U)$ and a complex sequence $\lambda_{j}, j \in \mathbb{N}$, such that $\sum_{j}\left|\lambda_{j}\right|<\infty$, such that

$$
\mathbf{f}(\varphi)=\sum_{j=0}^{\infty} \lambda_{j}\left\langle f_{j}, \varphi\right\rangle e_{j} .
$$

Since $\left\{f_{j} \mid j \in \mathbb{N}\right\}$ is an equicontinuous subset of $\mathcal{D}_{L^{1}}^{\prime(s)}(U)$, it is bounded and, by Proposition 2.4, there exist $h, C>0$ and $F_{j, \alpha} \in \mathcal{C}(\bar{U})$ such that

$$
f_{j}=\sum_{|\alpha|=0}^{\infty} D^{\alpha} F_{j, \alpha}
$$

and

$$
\sup _{j}\left(\sum_{\alpha \in \mathbb{N}^{d}} \frac{\alpha !^{p s}}{h^{|\alpha| p}}\left\|F_{j, \alpha}\right\|_{L^{\infty}(U)}^{p}\right)^{1 / p} \leq C .
$$

Define $\mathbf{F}_{\alpha}(x)=\sum_{j} \lambda_{j} F_{j, \alpha}(x) e_{j}$. To prove that $\mathbf{F}_{\alpha} \in \mathcal{C}(\bar{U} ; E)$, observe that, for each $j \in \mathbb{N}, \lambda_{j} F_{j, \alpha}(x) e_{j} \in \mathcal{C}(\bar{U} ; E)$ and the series $\sum_{j} \lambda_{j} F_{j, \alpha}(x) e_{j}$ converges absolutely in the $(B)$-space $\mathcal{C}(\bar{U} ; E)$. Hence, $\mathbf{F}_{\alpha} \in \mathcal{C}(\bar{U} ; E)$. Moreover,

$$
\begin{aligned}
\frac{\alpha !^{s}}{h^{|\alpha|}}\left\|\mathbf{F}_{\alpha}(x)\right\|_{E} & \leq \sum_{j=0}^{\infty}\left|\lambda_{j}\right| \frac{\alpha !^{s}}{h^{|\alpha|}}\left\|F_{j, \alpha}\right\|_{L^{\infty}(U)} \\
& \leq C \sum_{j=0}^{\infty}\left|\lambda_{j}\right|, \quad \text { for all } x \in \bar{U} .
\end{aligned}
$$

We obtain $\sup _{\alpha} \alpha !^{s} / h^{|\alpha|}\left\|\mathbf{F}_{\alpha}\right\|_{\mathcal{C}(\bar{U} ; E)}<\infty$. Since $U$ is bounded, (3.4) holds for any $h_{1}>h$. One easily verifies that the series $\sum_{j, \alpha} \lambda_{j}\left\langle D^{\alpha} F_{j, \alpha}, \varphi\right\rangle e_{j}$ converges absolutely in $E$ for each fixed $\varphi \in$ $\dot{\mathcal{B}}^{(s)}(U)$. Hence, $\mathbf{f}(\varphi)=\sum_{|\alpha|=0}^{\infty} D^{\alpha} \mathbf{F}_{\alpha}(\varphi)$, for each fixed $\varphi \in \dot{\mathcal{B}}^{(s)}(U)$. By Theorem 3.5, $\sum_{|\alpha|=0}^{\infty} D^{\alpha} \mathbf{F}_{\alpha}$ converges absolutely in $\mathcal{D}_{L^{1}}^{(s)}(U ; E)$; hence, $\mathbf{f}=\sum_{|\alpha|=0}^{\infty} D^{\alpha} \mathbf{F}_{\alpha}$. 
4. On the Cauchy problem in $\widetilde{\mathcal{D}}_{L^{p}, h}^{\prime s}(0, T ; E)$. In this section, $E$ is the $(B)$-space with the norm $\|\cdot\|$, and $D(A)$ is the domain of a closed linear operator $A$, endowed with the graph norm $\|u\|_{D(A)}=\|u\|+\|A u\|$. We use standard notation for the symbols $R(\lambda: A), \rho(A)$. The results obtained in previous sections will often be applied in the onedimensional case (i.e., $d=1$ ) when a bounded open set $U$ is equal to the interval $(0, T)$. In this case, we will use the more descriptive notations $L^{p}(0, T ; E), \mathcal{D}_{L^{p}, h}^{s}(0, T), \mathcal{D}_{L^{p}, h}^{(s)}(0, T), \dot{\mathcal{B}}^{(s)}(0, T), \mathcal{D}_{L^{p}, h}^{(s)}(0, T)$, $\mathcal{D}_{L^{1}}^{(s)}(0, T), \mathcal{D}_{L^{p}, h}^{(s)}(0, T ; E)$ and $\mathcal{D}_{L^{1}}^{(s)}(0, T ; E)$ for the spaces $L^{p}(U ; E)$, $\mathcal{D}_{L^{p}, h}^{s}(U), \mathcal{D}_{L^{p}, h}^{(s)}(U), \dot{\mathcal{B}}^{(s)}(U), \mathcal{D}_{L^{p}, h}^{(s)}(U), \mathcal{D}_{L^{1}}^{(s)}(U), \mathcal{D}_{L^{p}, h}^{(s)}(U ; E)$ and $\mathcal{D}_{L^{1}}^{(s)}(U ; E)$, respectively. Note that, by Sobolev imbedding theorem, every derivative of $\varphi \in \mathcal{D}_{L^{p}, h}^{s}(0, T)$ can be extended to uniformly continuous function on $[0, T]$. As in [6], we define the $E$-valued Sobolev space $W^{1, p}(0, T ; E)$ as the space of all $\mathbf{F}:[0, T] \rightarrow E$, such that

$$
\mathbf{F}(t)=F_{0}+\int_{0}^{t} \mathbf{F}^{\prime}(s) d s, \quad t \in[0, T],
$$

for some $F_{0} \in E$ and $\mathbf{F}^{\prime}(t) \in L^{p}(0, T ; E)$, with the norm $\|\mathbf{F}\|_{W^{1, p}(0, T: E)}=$ $\|\mathbf{F}\|_{L^{p}(0, T ; E)}+\left\|\mathbf{F}^{\prime}\right\|_{L^{p}(0, T ; E)}, 1 \leq p<\infty$. Observe that, if $\mathbf{F} \in$ $W^{1, p}(0, T ; E)$, then $\mathbf{F}$ is a continuous function with values in $E$ which is almost everywhere differentiable and its derivative is equal to $\mathbf{F}^{\prime}$ almost everywhere.

Let $1 \leq p<\infty$. Define $\widetilde{\mathcal{D}}_{L^{p}, h}^{\prime s}(0, T ; E)$ as a space of all sequences $\mathbf{f}=\left(\mathbf{F}_{\alpha}\right)_{\alpha}, \mathbf{F}_{\alpha} \in L^{p}(0, T ; E), \alpha \in \mathbb{N}$, such that

$$
\|\mathbf{f}\|_{\widetilde{\mathcal{D}}_{L^{p}, h}^{\prime s}(0, T ; E)}=\left(\sum_{\alpha \in \mathbb{N}} \frac{\alpha !^{p s}}{h^{p \alpha}}\left\|\mathbf{F}_{\alpha}\right\|_{L^{p}(0, T ; E)}^{p}\right)^{1 / p}<\infty .
$$

One easily verifies that it is a $(B)$-space with the norm (4.1). Each $\mathbf{f} \in \widetilde{\mathcal{D}}_{L^{p}, h}^{\prime s}(0, T ; E)$ generates an element of $\mathcal{L}\left(\mathcal{D}_{L^{q}, h}^{s}(0, T), E\right)$ by

$$
\langle\mathbf{f}, \varphi\rangle=\mathbf{f}(\varphi)=\sum_{\alpha \in \mathbb{N}}(-1)^{\alpha} \int_{0}^{T} \mathbf{F}_{\alpha}(t) \varphi^{(\alpha)}(t) d t \in E .
$$

Moreover, one easily verifies that the mapping $\mathbf{f} \mapsto\langle\mathbf{f}, \cdot\rangle, \widetilde{\mathcal{D}}_{L^{p}, h}^{\prime s}(0, T ; E) \rightarrow$ $\mathcal{L}_{b}\left(\mathcal{D}_{L^{q}, h}^{s}(0, T), E\right)$ is continuous. 
Remark 4.1. It is worth noting that this mapping is not injective. To see this, let $\psi \in \mathcal{D}^{(s)}(0, T), \psi \neq 0$. Take a nonzero element $e$ of $E$ and define $\mathbf{F}(x)=\psi^{\prime}(x) e$ and $\mathbf{G}(x)=\psi(x) e, x \in(0, T)$. Obviously, $\mathbf{F}, \mathbf{G} \in L^{p}(0, T ; E)$, for any $1 \leq p \leq \infty$. Define $\mathbf{f}, \mathbf{g} \in \widetilde{\mathcal{D}}_{L^{p}, h}^{\prime}(0, T ; E)$ by $\mathbf{f}=(\mathbf{F}, 0,0, \ldots)$ and $\mathbf{g}=(0, \mathbf{G}, 0, \ldots)$. Observe that, for $\varphi \in$ $\mathcal{D}_{L^{q}, h}^{s}(0, T)$,

$$
\begin{aligned}
\langle\mathbf{f}, \varphi\rangle & =e \int_{0}^{T} \psi^{\prime}(x) \varphi(x) d x \\
& =-e \int_{0}^{T} \psi(x) \varphi^{\prime}(x) d x=\langle\mathbf{g}, \varphi\rangle .
\end{aligned}
$$

Hence, $\langle\mathbf{f}, \cdot\rangle$ and $\langle\mathbf{g}, \cdot\rangle$ are the same element of $\mathcal{L}_{b}\left(\mathcal{D}_{L^{q}, h}^{s}(0, T), E\right)$.

Note that $L^{p}(0, T ; E)$ can be continuously imbedded in $\widetilde{\mathcal{D}}_{L^{p}, h}^{\prime s}(0, T ; E)$ by $\mathbf{F} \mapsto(\mathbf{F}, 0,0, \ldots)$.

Let $1 \leq p<\infty$. We define $\widetilde{\mathcal{D}}_{W^{1, p}, h}^{\prime}(0, T ; E)$ as the space of all sequences $\mathbf{f}=\left(\mathbf{F}_{\alpha}\right)_{\alpha}$, where $\mathbf{F}_{\alpha} \in W^{1, p}(0, T ; E)$ and

$$
\begin{aligned}
\|\mathbf{f}\|_{\widetilde{\mathcal{D}}_{W^{1, p}, h}^{\prime s}(0, T ; E)} & =\left(\sum_{\alpha \in \mathbb{N}} \frac{\alpha !^{p s}}{h^{p \alpha}}\left(\left\|\mathbf{F}_{\alpha}\right\|_{L^{p}(0, T ; E)}^{p}+\left\|\mathbf{F}_{\alpha}^{\prime}\right\|_{L^{p}(0, T ; E)}^{p}\right)\right)^{1 / p} \\
& <\infty
\end{aligned}
$$

Equipped with the norm $\|\cdot\|_{\widetilde{\mathcal{D}}_{W^{1, p}, h}^{\prime}(0, T ; E)}$, it becomes a $(B)$-space.

$\widetilde{\mathcal{D}}_{W^{1, p}, h}^{\prime s}(0, T ; E)$ is continuously injected into $\widetilde{\mathcal{D}}_{L^{p}, h}^{\prime s}(0, T ; E)$. For $\mathbf{f}=\left(\mathbf{F}_{\alpha}\right)_{\alpha} \in \widetilde{\mathcal{D}}_{W^{1, p}, h}^{\prime s}(0, T ; E), \mathbf{f}^{\prime}=\widetilde{\mathbf{f}}=\left(\widetilde{\mathbf{F}}_{\alpha}\right)_{\alpha} \in \widetilde{\mathcal{D}}_{L^{p}, h}^{\prime s}(0, T ; E)$, where $\widetilde{\mathbf{F}}_{\alpha}=\mathbf{F}_{\alpha}^{\prime}$ is the classical derivative almost everywhere in $(0, T)$. Moreover, the mapping $\mathbf{f} \mapsto \mathbf{f}^{\prime}, \widetilde{\mathcal{D}}_{W^{1, p}, h}^{\prime s}(0, T ; E) \rightarrow \widetilde{\mathcal{D}}_{L^{p}, h}^{\prime s}(0, T ; E)$, is continuous.

Our main assumption is that the Hille-Yosida condition holds for the resolvent of the operator $A$ :

$$
\left\|(\lambda-\omega)^{k} R(\lambda: A)^{k}\right\| \leq C, \quad \text { for } \lambda>\omega, k \in \mathbb{Z}_{+} .
$$

From now on, we will always denote these constants by $\omega$ and $C$. 
4.1. Various types of solutions. We need the following technical lemma.

Lemma 4.2. Let $1 \leq p<\infty$ and $\mathbf{g}=\left(\mathbf{G}_{\alpha}\right)_{\alpha} \in \widetilde{\mathcal{D}}_{L^{p}, h}^{s}(0, T ; D(A))$. Then, for every $\varphi \in \mathcal{D}_{L^{q}}^{s}(0, T),\langle\mathbf{g}, \varphi\rangle \in D(A)$ and

$$
A \sum_{\alpha=0}^{\infty}(-1)^{\alpha} \int_{0}^{T} \mathbf{G}_{\alpha}(t) \varphi^{(\alpha)}(t) d t=\sum_{\alpha=0}^{\infty}(-1)^{\alpha} \int_{0}^{T} A \mathbf{G}_{\alpha}(t) \varphi^{(\alpha)}(t) d t .
$$

Proof. First observe that, for each $\alpha \in \mathbb{N}, \mathbf{G}_{\alpha} \varphi^{(\alpha)} \in L^{1}(0, T ; D(A))$ and $A \mathbf{G}_{\alpha} \varphi^{(\alpha)} \in L^{1}(0, T ; E)$ since $\mathbf{G}_{\alpha}(t) \in L^{p}(0, T ; D(A))$ and $\varphi \in$ $\mathcal{D}_{L^{q}}^{s}(0, T)$. Then

$$
A \int_{0}^{T} \mathbf{G}_{\alpha}(t) \varphi^{(\alpha)}(t) d t=\int_{0}^{T} A \mathbf{G}_{\alpha}(t) \varphi^{(\alpha)}(t) d t .
$$

Moreover, observe that

$$
\sum_{\alpha=0}^{\infty}\left\|\int_{0}^{T} \mathbf{G}_{\alpha}(t) \varphi^{(\alpha)}(t) d t\right\|_{D(A)} \leq\left\|\left(\mathbf{G}_{\alpha}\right)_{\alpha}\right\|_{\widetilde{\mathcal{D}}_{L^{p}, h}^{\prime s}(0, T ; D(A))}\|\varphi\|_{\mathcal{D}_{L^{q}}^{s}(0, T)} .
$$

We obtain that

$$
\sum_{\alpha=0}^{\infty}(-1)^{\alpha} \int_{0}^{T} \mathbf{G}_{\alpha}(t) \varphi^{(\alpha)}(t) d t
$$

converges absolutely in $D(A)$, i.e., $\langle\mathbf{g}, \varphi\rangle \in D(A)$. Hence,

$$
A \sum_{\alpha=0}^{\infty}(-1)^{\alpha} \int_{0}^{T} \mathbf{G}_{\alpha}(t) \varphi^{(\alpha)}(t) d t=\sum_{\alpha=0}^{\infty}(-1)^{\alpha} A \int_{0}^{T} \mathbf{G}_{\alpha}(t) \varphi^{(\alpha)}(t) d t,
$$

which, together with (4.3), completes the proof of the lemma.

Let $u_{0, \alpha} \in E, \alpha \in \mathbb{N}$, be such that

$$
\left(\sum_{\alpha=0}^{\infty} \frac{\alpha !^{p s}}{h^{p \alpha}}\left\|u_{0, \alpha}\right\|_{E}^{p}\right)^{1 / p}<\infty .
$$

Then the constant functions $\widetilde{\mathbf{U}}_{\alpha}(t)=u_{0, \alpha}, t \in[0, T]$, are such that $\widetilde{\mathbf{U}}_{\alpha} \in L^{p}(0, T ; E)$ and (4.1) holds. Hence, $\left(\widetilde{\mathbf{U}}_{\alpha}\right)_{\alpha} \in \widetilde{\mathcal{D}}_{L^{p}, h}^{\prime s}(0, T ; E)$. In the sequel, if $u_{0, \alpha}, \alpha \in \mathbb{N}$, are such elements, we will denote the corresponding constant functions simply by $u_{0, \alpha}$ and the element 
$\left(u_{0, \alpha}\right)_{\alpha}$ of $\widetilde{\mathcal{D}}_{L^{p}, h}^{\prime s}(0, T ; E)$ that they generate by $u_{0}$. We also use the notation $\left\|u_{0}\right\|_{\widetilde{\mathcal{D}}_{L^{p}, h}^{\prime s}(0, T ; E)}$ for the norm of this element of $\widetilde{\mathcal{D}}_{L^{p}, h}^{s}(0, T ; E)$.

We recall from [6] the definition of two types of solutions of the Cauchy problem (0.1) (here they are restated to fit in our setting). We also define a weak version of them. Let $A: D(A) \subseteq E \rightarrow E$ be a closed linear operator in the $(B)$-space $E, \mathbf{f} \in \widetilde{\mathcal{D}}_{L^{p}, h}^{s}(0, T ; E)$ and $u_{0, \alpha} \in E$, $\alpha \in \mathbb{N}$.

1. We say that $\mathbf{u}=\left(\mathbf{U}_{\alpha}\right)_{\alpha}$ is a strict solution, respectively, strict weak solution, in $\widetilde{\mathcal{D}}_{L^{p}, h}^{\prime s}(0, T ; E)$ of $(0.1)$ if $\mathbf{u} \in \widetilde{\mathcal{D}}_{W^{1, p}, h}^{\prime s}(0, T ; E) \cap$ $\widetilde{\mathcal{D}}_{L^{p}, h}^{\prime s}(0, T ; D(A))$ and

$$
\mathbf{U}_{\alpha}^{\prime}(t)=A \mathbf{U}_{\alpha}(t)+\mathbf{F}_{\alpha}(t), \quad t \in[0, T] \text { almost everywhere }
$$

and

$$
\mathbf{U}_{\alpha}(0)=u_{0, \alpha}, \quad \text { for all } \alpha \in \mathbb{N},
$$

respectively, for each $\varphi \in \mathcal{D}_{L^{q}, h}^{s}(0, T)$ satisfy

$$
\left\langle\mathbf{u}^{\prime}(t), \varphi(t)\right\rangle=A\langle\mathbf{u}(t), \varphi(t)\rangle+\langle\mathbf{f}(t), \varphi(t)\rangle
$$

and

$$
\mathbf{U}_{\alpha}(0)=u_{0, \alpha}, \quad \text { for all } \alpha \in \mathbb{N} .
$$

We know by Lemma 4.2 that $\langle\mathbf{u}(t), \varphi(t)\rangle \in D(A)$ for each $\varphi \in$ $\mathcal{D}_{L^{q}, h}^{s}(0, T)$. Also, note that in both cases (of strict or of strict weak solution of (0.1)) we have

$$
\left\|u_{0, \alpha}\right\|_{E}^{p} \leq 2^{p} T^{-1}\left\|\mathbf{U}_{\alpha}\right\|_{L^{p}(0, T ; E)}+2^{p} T^{p / q}\left\|\mathbf{U}_{\alpha}^{\prime}\right\|_{L^{p}(0, T ; E)} .
$$

Hence, $u_{0}=\left(u_{0, \alpha}\right)_{\alpha}$ satisfies (4.4).

2. We say that $\mathbf{u} \in \widetilde{\mathcal{D}}_{L^{p}, h}^{\prime s}(0, T ; E)$ is an $F$-solution, respectively, $F$-weak solution in $\widetilde{\mathcal{D}}_{L^{p}, h}^{\prime s}(0, T ; E)$ of $(0.1)$ if, for every $k \in \mathbb{N}$, there is $\mathbf{u}_{k}=\left(\mathbf{U}_{k, \alpha}\right)_{\alpha} \in \widetilde{\mathcal{D}}_{W^{1, p}, h}^{\prime s}(0, T ; E) \cap \widetilde{\mathcal{D}}_{L^{p}, h}^{\prime s}(0, T ; D(A))$ such that from

$$
\mathbf{U}_{k, \alpha}^{\prime}(t)=A \mathbf{U}_{k, \alpha}(t)+\mathbf{F}_{k, \alpha}(t), \quad t \in[0, T] \text { almost everywhere }
$$

and

$$
\mathbf{U}_{k, \alpha}(0)=u_{0, k, \alpha},
$$


we have

$$
\begin{aligned}
\lim _{k \rightarrow \infty}\left(\left\|\mathbf{u}_{k}-\mathbf{u}\right\|_{\widetilde{\mathcal{D}}_{L^{p}, h}^{\prime s}(0, T ; E)}+\left\|\mathbf{f}_{k}-\mathbf{f}\right\|_{\widetilde{\mathcal{D}}_{L^{p}, h}^{\prime s}(0, T ; E)}\right. & \\
& \left.+\left\|u_{0, k}-u_{0}\right\|_{\widetilde{\mathcal{D}}_{L^{p}, h}^{\prime s}(0, T ; E)}\right)=0
\end{aligned}
$$

respectively, from

$$
\left\langle\mathbf{u}_{k}^{\prime}(t), \varphi(t)\right\rangle=A\left\langle\mathbf{u}_{k}(t), \varphi(t)\right\rangle+\left\langle\mathbf{f}_{k}(t), \varphi(t)\right\rangle, \quad \text { for all } \varphi \in \mathcal{D}_{L^{q}, h}^{s}(0, T)
$$

and

$$
\mathbf{U}_{k, \alpha}(0)=u_{0, k, \alpha}, \quad \text { for all } k, \alpha \in \mathbb{N}
$$

we have that, for every $\varphi \in \mathcal{D}_{L^{q}, h}^{s}(0, T)$,

(4.6) $\lim _{k \rightarrow \infty}\left(\left\|\left\langle\mathbf{u}_{k}-\mathbf{u}, \varphi\right\rangle\right\|_{E}+\left\|\left\langle\mathbf{f}_{k}-\mathbf{f}, \varphi\right\rangle\right\|_{E}+\left\|\left\langle u_{0, k}-u_{0}, \varphi\right\rangle\right\|_{E}\right)=0$.

From the above definitions, it is clear that a strict, respectively a strict weak solution, in $\widetilde{\mathcal{D}}_{L^{p}, h}^{\prime s}(0, T ; E)$ is an $F$-solution, respectively $F$-weak solution, in $\widetilde{\mathcal{D}}_{L^{p}, h}^{\prime s}(0, T ; E)$.

Remark 4.3. If a strict weak solution of $(0.1)$ in $\widetilde{\mathcal{D}}_{L^{p}, h}^{\prime s}(0, T ; E)$ exists, then it is not unique. To see this, let $\psi \in \mathcal{D}^{(s)}(0, T)$ and $e \in D(A)$ be such that $\psi \neq 0$ and $e \neq 0$. Define $\mathbf{v}=\left(\mathbf{V}_{\alpha}\right)_{\alpha} \in \widetilde{\mathcal{D}}_{L^{p}, h}^{\prime}(0, T ; E)$ by $\mathbf{V}_{0}(t)=\psi^{\prime}(t) e, \mathbf{V}_{1}(t)=-\psi(t) e$ and $\mathbf{V}_{\alpha}(t)=0$, for $\alpha \geq 2, \alpha \in \mathbb{N}$. Obviously $\mathbf{v} \in \widetilde{\mathcal{D}}_{W^{1, p}, h}^{\prime s}(0, T ; E) \cap \widetilde{\mathcal{D}}_{L^{p}, h}^{\prime s}(0, T ; D(A))$ and $\overline{\mathbf{V}}_{\alpha}(0)=0$, for all $\alpha \in \mathbb{N}$. Moreover, it is easy to verify that the operators $\langle\mathbf{v}, \cdot\rangle,\left\langle\mathbf{v}^{\prime}, \cdot\right\rangle \in \mathcal{L}\left(\mathcal{D}_{L^{q}, h}^{s}(0, T), E\right)$ are, in fact, the zero operator. Hence, if $\mathbf{u}$ is a strict weak solution of $(0.1)$ in $\widetilde{\mathcal{D}}_{L^{p}, h}^{\prime s}(0, T ; E)$, then so is $\mathbf{u}+\mathbf{v}$.

One can use the same construction to prove that the $F$-weak solution in $\widetilde{\mathcal{D}}_{L^{p}, h}^{\prime s}(0, T ; E)$ of $(0.1)$ is also not unique.

4.2. The existence of solutions. Now we consider the existence of such solutions of the Cauchy problem (0.1).

Proposition 4.4. If $\mathbf{u}$ is a strict, respectively an F-solution, of the Cauchy problem (0.1), then it is also strict weak, respectively $F$-weak solution, of (0.1). 
Proof. The proof follows from Lemma 4.2 and the fact that the mapping $\mathbf{g} \mapsto\langle\mathbf{g}, \cdot\rangle, \widetilde{\mathcal{D}}_{L^{p}, h}^{\prime s}(0, T ; E) \rightarrow \mathcal{L}_{b}\left(\mathcal{D}_{L^{q}, h}^{s}(0, T), E\right)$ is continuous.

The proof of the next theorem relies heavily on the results obtained in [6]. The parts in brackets are consequences of Proposition 4.4.

\section{Theorem 4.5.}

(i) The Cauchy problem (0.1) has an F-solution (respectively an F-weak solution) in $\widetilde{\mathcal{D}}_{L^{p}, h}^{\prime s}(0, T ; E)$ for every $\mathbf{f}=\left(\mathbf{F}_{\alpha}\right)_{\alpha} \in$ $\widetilde{\mathcal{D}}_{L^{p}, h}^{\prime s}(0, T ; E)$ and $u_{0}=\left(u_{0, \alpha}\right)_{\alpha}$ such that $\left(u_{0, \alpha}\right)_{\alpha}$ satisfies $(4.4)$ and $u_{0, \alpha} \in \overline{D(A)}$, for all $\alpha \in \mathbb{N}$. In the case of F-solution, it is unique.

(ii) The Cauchy problem (0.1) has a strict solution (respectively a strict weak solution) in $\widetilde{\mathcal{D}}_{L^{p}, h}^{\prime s}(0, T ; E)$ for every $\mathbf{f}=\left(\mathbf{F}_{\alpha}\right)_{\alpha} \in$ $\widetilde{\mathcal{D}}_{W^{1, p}, h}^{\prime}(0, T ; E)$ and $u_{0}=\left(u_{0, \alpha}\right)_{\alpha}$ such that $u_{0, \alpha} \in D(A)$ and $A u_{0, \alpha}+\mathbf{F}_{\alpha}(0) \in \overline{D(A)}$, for all $\alpha \in \mathbb{N}$ and $\left(u_{0, \alpha}\right)_{\alpha}$, and $\left(A u_{0, \alpha}\right)_{\alpha}$ satisfies (4.4). In the case of a strict solution, it is unique.

Proof. First, we will prove (i). By [6, Theorem 7.2] (see also [6, Appendix]) for each fixed $\alpha \in \mathbb{N}$, the problem $\mathbf{U}_{\alpha}^{\prime}=A \mathbf{U}_{\alpha}+\mathbf{F}_{\alpha}$, $\mathbf{U}_{\alpha}(0)=u_{0, \alpha}$ has an $F$-solution in $L^{p}(0, T ; E)$. In other words, there exist $\mathbf{U}_{k, \alpha} \in W^{1, p}(0, T ; E) \cap L^{p}(0, T ; D(A)), \mathbf{F}_{k, \alpha} \in L^{p}(0, T ; E)$, $u_{0, k, \alpha} \in E$ and $k \in \mathbb{Z}_{+}$, such that $\mathbf{U}_{k, \alpha}^{\prime}=A \mathbf{U}_{k, \alpha}+\mathbf{F}_{k, \alpha}, \mathbf{U}_{k, \alpha}(0)=$ $u_{0, k, \alpha}$ and

$$
\begin{aligned}
\lim _{k \rightarrow \infty}\left(\left\|\mathbf{U}_{k, \alpha}-\mathbf{U}_{\alpha}\right\|_{L^{p}(0, T ; E)}+\| \mathbf{F}_{k, \alpha}\right. & -\mathbf{F}_{\alpha} \|_{L^{p}(0, T ; E)} \\
& \left.+\left\|u_{0, k, \alpha}-u_{0, \alpha}\right\|_{E}\right)=0 .
\end{aligned}
$$

Moreover, by [6, Theorem 5.1] (see also [6, Theorem A.1, Appendix]), each $\mathbf{U}_{\alpha}$ is in fact in $\mathcal{C}(0, T ; E), \mathbf{U}_{\alpha}(t) \in \overline{D(A)}$, for all $t \in[0, T]$, $\mathbf{U}_{\alpha}(0)=u_{0, \alpha}$ and

$$
\left\|\mathbf{U}_{\alpha}(t)\right\| \leq C e^{\omega t}\left(\left\|\mathbf{U}_{\alpha}(0)\right\|+\int_{0}^{t} e^{-\omega s}\left\|\mathbf{F}_{\alpha}(s)\right\| d s\right), \quad t \in[0, T] .
$$


Using this estimate, one easily verifies that $\mathbf{u}=\left(\mathbf{U}_{\alpha}\right)_{\alpha} \in \widetilde{\mathcal{D}}_{L^{p}, h}^{\prime s}(0, T ; E)$. We will prove that this is an $(F)$-solution of $(0.1)$.

Let $k \in \mathbb{Z}_{+}$. Take $n_{k} \in \mathbb{Z}_{+}$such that

$$
\begin{aligned}
\sum_{\alpha=n_{k}}^{\infty} \frac{\alpha !^{p s}}{h^{p \alpha}}\left\|\mathbf{F}_{\alpha}\right\|_{L^{p}(0, T ; E)}^{p} & \leq \frac{1}{(2 k)^{p}}, \\
\sum_{\alpha=n_{k}}^{\infty} \frac{\alpha !^{p s}}{h^{p \alpha}}\left\|\mathbf{U}_{\alpha}\right\|_{L^{p}(0, T ; E)}^{p} & \leq \frac{1}{(2 k)^{p}}
\end{aligned}
$$

and

$$
\sum_{\alpha=n_{k}}^{\infty} \frac{\alpha !^{p s}}{h^{p \alpha}}\left\|u_{0, \alpha}\right\|_{E}^{p} \leq \frac{1}{(2 k)^{p}} .
$$

For each $0 \leq \alpha \leq n_{k}-1$, by (4.7) we can take $\mathbf{F}_{k_{\alpha}, \alpha}, \mathbf{U}_{k_{\alpha}, \alpha}$ and $u_{0, k_{\alpha}, \alpha}$ such that

$$
\begin{aligned}
\sum_{\alpha=0}^{n_{k}-1} \frac{\alpha !^{p s}}{h^{p \alpha}}\left(\left\|\mathbf{U}_{k_{\alpha}, \alpha}-\mathbf{U}_{\alpha}\right\|_{L^{p}(0, T ; E)}^{p}\right. & +\left\|\mathbf{F}_{k_{\alpha}, \alpha}-\mathbf{F}_{\alpha}\right\|_{L^{p}(0, T ; E)}^{p} \\
& \left.+\left\|u_{0, k_{\alpha}, \alpha}-u_{0, \alpha}\right\|_{E}^{p}\right) \leq \frac{1}{(2 k)^{p}}
\end{aligned}
$$

and

$$
\mathbf{U}_{k_{\alpha}, \alpha}^{\prime}=A \mathbf{U}_{k_{\alpha}, \alpha}+\mathbf{F}_{k_{\alpha}, \alpha}, \quad \mathbf{U}_{k_{\alpha}, \alpha}(0)=u_{0, k_{\alpha}, \alpha} .
$$

For $0 \leq \alpha \leq n_{k}-1$, define

$$
\mathbf{V}_{k, \alpha}=\mathbf{U}_{k_{\alpha}, \alpha}, \quad v_{0, k, \alpha}=u_{0, k_{\alpha}, \alpha}
$$

and

$$
\mathbf{G}_{k, \alpha}=\mathbf{F}_{k_{\alpha}, \alpha} .
$$

For $\alpha \geq n_{k}$, put

$$
\mathbf{V}_{k, \alpha}=0, \quad v_{0, k, \alpha}=0 \quad \text { and } \quad \mathbf{G}_{k, \alpha}=0 .
$$

Then

$$
\begin{aligned}
& \mathbf{v}_{k}=\left(\mathbf{V}_{k, \alpha}\right)_{\alpha} \in \widetilde{\mathcal{D}}_{W^{1, p}, h}^{\prime s}(0, T ; E) \cap \widetilde{\mathcal{D}}_{L^{p}, h}^{\prime s}(0, T ; D(A)), \\
& \mathbf{g}_{k}=\left(\mathbf{G}_{k, \alpha}\right)_{\alpha} \in \widetilde{\mathcal{D}}_{L^{p}, h}^{s}(0, T ; E)
\end{aligned}
$$


and $v_{0, k}=\left(v_{0, k, \alpha}\right)_{\alpha}$ is such that

$$
\sum_{\alpha=0}^{\infty} \frac{(\alpha !)^{p s}}{h^{p \alpha}}\left\|v_{0, k, \alpha}\right\|_{E}^{p}<\infty
$$

Also, $\mathbf{v}_{k}(0)=v_{0, k}$. By definition, we have $\mathbf{V}_{k, \alpha}^{\prime}=A \mathbf{V}_{k, \alpha}+\mathbf{G}_{k, \alpha}$ for all $\alpha \in \mathbb{N}$. We will prove that $\mathbf{v}_{k} \rightarrow \mathbf{u}, \mathbf{g}_{k} \rightarrow \mathbf{f}$ and $v_{0, k} \rightarrow u_{0}$ in $\widetilde{\mathcal{D}}_{L^{p}, h}^{\prime s}(0, T ; E)$; hence, $\mathbf{u}$ is an $F$-solution of $(0.1)$.

Let $\varepsilon>0$. Take $k_{0} \in \mathbb{Z}_{+}$such that $1 / k_{0} \leq \varepsilon$. For $k \geq k_{0}, k \in \mathbb{Z}_{+}$, we have

$$
\begin{aligned}
& \left\|\mathbf{v}_{k}-\mathbf{u}\right\|_{\widetilde{\mathcal{D}}_{L^{\prime}, h}^{f^{\prime}}(0, T ; E)}^{p} \\
& =\sum_{\alpha=0}^{n_{k}-1} \frac{\alpha !^{p s}}{h^{p \alpha}}\left\|\mathbf{V}_{k, \alpha}-\mathbf{U}_{\alpha}\right\|_{L^{p}(0, T ; E)}^{p}+\sum_{\alpha=n_{k}}^{\infty} \frac{\alpha !^{p s}}{h^{p \alpha}}\left\|\mathbf{U}_{\alpha}\right\|_{L^{p}(0, T ; E)}^{p} \\
& \leq \sum_{\alpha=0}^{n_{k}-1} \frac{\alpha !^{p s}}{h^{p \alpha}}\left\|\mathbf{U}_{k_{\alpha}, \alpha}-\mathbf{U}_{\alpha}\right\|_{L^{p}(0, T ; E)}^{p}+\frac{\varepsilon^{p}}{2^{p}} \leq \frac{2 \varepsilon^{p}}{2^{p}}
\end{aligned}
$$

Hence, $\left\|\mathbf{v}_{k}-\mathbf{u}\right\|_{\widetilde{\mathcal{D}}_{L^{p}, h}^{\prime s}(0, T ; E)} \leq \varepsilon$. Similarly,

$$
\left\|\mathbf{g}_{k}-\mathbf{f}\right\|_{\widetilde{\mathcal{D}}_{L^{p}, h}^{\prime s}(0, T ; E)} \leq \varepsilon
$$

and

$$
\left(\sum_{\alpha=0}^{\infty} \frac{\alpha !^{p s}}{h^{p \alpha}}\left\|v_{0, k, \alpha}-u_{0, \alpha}\right\|_{L^{p}(0, T ; E)}^{p}\right)^{1 / p} \leq \varepsilon, \quad \text { for } k \geq k_{0} .
$$

It remains to prove the uniqueness. If $\widetilde{\mathbf{u}}=\left(\widetilde{\mathbf{U}}_{\alpha}\right)_{\alpha} \in \widetilde{\mathcal{D}}_{L^{p}, h}^{\prime s}(0, T ; E)$ is another $F$-solution of $(0.1)$, then $\widetilde{\mathbf{U}}_{\alpha}$ is an $F$-solution to the problem $\widetilde{\mathbf{U}}_{\alpha}^{\prime}(t)=A \widetilde{\mathbf{U}}_{\alpha}(t)+\mathbf{F}_{\alpha}(t), \widetilde{\mathbf{U}}_{\alpha}(0)=u_{0, \alpha}$, for each $\alpha \in \mathbb{N}$. But, [6, Theorem 5.1] (see also [6, Theorem A.1, Appendix]) implies that the $F$-solution to this problem must be unique; hence, $\widetilde{\mathbf{U}}_{\alpha}=\mathbf{U}_{\alpha}$, which proves the desired uniqueness.

To prove (ii), observe that [6, Theorem 8.1] (see also [6, Theorem A.2, Appendix]) implies that, for each $\alpha \in \mathbb{N}$, there exists $\mathbf{U}_{\alpha} \in$ $\mathcal{C}^{1}(0, T ; E) \cap \mathcal{C}(0, T ; D(A))$ such that

$$
\mathbf{U}_{\alpha}^{\prime}(t)=A \mathbf{U}_{\alpha}(t)+\mathbf{F}_{\alpha}(t), \quad \text { for all } t \in[0, T] \text { and } \mathbf{U}_{\alpha}(0)=u_{0, \alpha}
$$


and it satisfies (4.8) and

$$
\begin{aligned}
\left\|\mathbf{U}_{\alpha}^{\prime}(t)\right\| \leq & C e^{\omega t}\left(\left\|A u_{0, \alpha}+\mathbf{F}_{\alpha}(0)\right\|\right. \\
& \left.+\int_{0}^{t} e^{-\omega s}\left\|\mathbf{F}_{\alpha}^{\prime}(s)\right\| d s\right), \quad t \in[0, T] .
\end{aligned}
$$

Moreover, by (4.9) and (4.10), we have

$$
\begin{aligned}
\left\|A \mathbf{U}_{\alpha}(t)\right\| \leq & C e^{2|\omega| T}\left(\left\|A u_{0, \alpha}\right\|+\left\|\mathbf{F}_{\alpha}(0)\right\|+T^{1 / q}\left\|\mathbf{F}_{\alpha}^{\prime}\right\|_{L^{p}(0, T ; E)}\right) \\
& +\left\|\mathbf{F}_{\alpha}(t)\right\|, \quad t \in[0, T] .
\end{aligned}
$$

Since $\mathbf{f} \in \widetilde{\mathcal{D}}_{W^{1, p}, h}^{\prime s}(0, T ; E),\left(u_{0, \alpha}\right)_{\alpha}$ and $\left(A u_{0, \alpha}\right)_{\alpha}$ satisfy (4.4), by the above estimate, (4.8) and (4.10), we can conclude

$$
\mathbf{u}=\left(\mathbf{U}_{\alpha}\right)_{\alpha} \in \widetilde{\mathcal{D}}_{W^{1, p}, h}^{s}(0, T ; E) \cap \widetilde{\mathcal{D}}_{L^{p}, h}^{\prime s}(0, T ; D(A)) .
$$

Hence, $\mathbf{u}$ is a strict solution. The uniqueness follows from [6, Theorem 8.1] (see also [6, Theorem A.2, Appendix]) by similar arguments as in (i).

By following the proof of [8, Theorem 2.5], one can prove Theorem 4.5 by using locally Lipschitz continuous integrated semigroups.

4.3. Solutions in $\mathcal{D}_{L^{1}}^{\prime(s)}(0, T ; E)$. Let $\mathbf{g} \in \mathcal{D}_{L^{1}}^{(s)}(0, T ; E)$. By Theorem 3.6 for $1<p<\infty$, there exist $h_{1}>0$ and $\mathbf{G}_{\alpha} \in L^{p}(0, T ; E)$, $\alpha \in \mathbb{N}$, such that

$$
\sum_{\alpha=0}^{\infty} \frac{\alpha !^{p s}}{h_{1}^{p \alpha}}\left\|\mathbf{G}_{\alpha}\right\|_{L^{p}(0, T ; E)}^{p}<\infty \quad \text { and } \quad \mathbf{g}=\sum_{\alpha=0}^{\infty} \mathbf{G}_{\alpha}^{(\alpha)} .
$$

For the moment, for $\mathbf{g} \in \mathcal{D}_{L^{1}}^{\prime(s)}(0, T ; E)=\mathcal{L}_{b}\left(\dot{\mathcal{B}}^{(s)}(0, T), E\right)$, denote by $\mathbf{g}(\varphi)$ the action of $\mathbf{g}$ on $\varphi \in \dot{\mathcal{B}}^{(s)}(0, T)$. On the other hand, put $\widetilde{\mathrm{g}}=\left(\mathbf{G}_{\alpha}\right)_{\alpha} \in \widetilde{\mathcal{D}}_{L^{p}, h}^{\prime s}(0, T ; E)$. By the way we define the operator $\langle\widetilde{\mathbf{g}}, \cdot\rangle \in \mathcal{L}_{b}\left(\mathcal{D}_{L^{q}, h}^{(s)}(0, T), E\right)$, one easily verifies that $\mathbf{g}(\varphi)=\langle\widetilde{\mathbf{g}}, \varphi\rangle$ for all $\varphi \in \dot{\mathcal{B}}^{(s)}(0, T) \subseteq \mathcal{D}_{L^{q}, h}^{(s)}(0, T)$. Hence, if $\mathbf{g} \in \mathcal{D}_{L^{1}}^{(s)}(0, T ; E)$ has the representation (4.11), we will denote by $\langle\mathbf{g}, \cdot\rangle$ the action $\mathbf{g}(\cdot)$. 
Let $\mathbf{g} \in \mathcal{D}_{L^{1}}^{\prime(s)}(0, T ; E)$ have the representation (4.11). Define $\widetilde{\mathbf{G}}_{0}=0$ and

$$
\widetilde{\mathbf{G}}_{\alpha}(t)=\int_{0}^{t} \mathbf{G}_{\alpha-1}(s) d s, \quad t \in[0, T]
$$

for $\alpha \in \mathbb{Z}_{+}$. Then, obviously, $\widetilde{\mathbf{G}}_{\alpha} \in W^{1, p}(0, T ; E), \widetilde{\mathbf{G}}_{\alpha}(0)=0$ and $\widetilde{\mathbf{G}}_{\alpha}^{\prime}=\mathbf{G}_{\alpha-1}$ almost everywhere for all $\alpha \in \mathbb{Z}_{+}$, and if we put $h>h_{1}$, we have

$$
\sum_{\alpha=0}^{\infty} \frac{\alpha !^{p s}}{h^{p \alpha}}\left(\left\|\widetilde{\mathbf{G}}_{\alpha}\right\|_{L^{p}(0, T ; E)}^{p}+\left\|\widetilde{\mathbf{G}}_{\alpha}^{\prime}\right\|_{L^{p}(0, T ; E)}^{p}\right)<\infty .
$$

By Theorem 3.6,

$$
\sum_{\alpha=1}^{\infty} \widetilde{\mathbf{G}}_{\alpha}^{(\alpha)} \in \mathcal{D}_{L^{1}}^{(s)}(0, T ; E) .
$$

Also, for $\varphi \in \dot{\mathcal{B}}^{(s)}(0, T)$,

$$
\begin{aligned}
& \sum_{\alpha=1}^{\infty}(-1)^{\alpha} \int_{0}^{T} \widetilde{\mathbf{G}}_{\alpha}(t) \varphi^{(\alpha)}(t) d t \\
& =\sum_{\alpha=0}^{\infty}(-1)^{\alpha} \int_{0}^{T} \widetilde{\mathbf{G}}_{\alpha+1}^{\prime}(t) \varphi^{(\alpha)}(t) d t \\
& =\sum_{\alpha=0}^{\infty}(-1)^{\alpha} \int_{0}^{T} \mathbf{G}_{\alpha}(t) \varphi^{(\alpha)}(t) d t \\
& =\langle\mathbf{g}, \varphi\rangle,
\end{aligned}
$$

i.e., $\mathbf{g}=\sum_{\alpha=1}^{\infty} \widetilde{\mathbf{G}}_{\alpha}^{(\alpha)}$. In other words, for $\mathbf{g} \in \mathcal{D}_{L^{1}}^{(s)}(0, T ; E)$ and $1<p<\infty$, we can always find $h>0$ such that

$$
\mathbf{g}=\sum_{\alpha=0}^{\infty} \widetilde{\mathbf{G}}_{\alpha}^{(\alpha)},
$$

where

$$
\widetilde{\mathbf{G}}_{\alpha} \in W^{1, p}(0, T ; E), \quad \widetilde{\mathbf{G}}_{\alpha}(0)=0, \quad \alpha \in \mathbb{N},
$$

such that (4.12) holds. Moreover, in this notation, if we put

$$
\widetilde{\mathbf{f}}=\left(\widetilde{\mathbf{G}}_{\alpha}^{\prime}\right)_{\alpha} \in \widetilde{\mathcal{D}}_{L^{p}, h}^{s}(0, T ; E),
$$


then $\langle\widetilde{\mathbf{f}}, \cdot\rangle$ and the $E$-valued ultradistribution $\mathbf{g}^{\prime} \in \mathcal{D}_{L^{1}}^{\prime(s)}(0, T ; E)$ (where $\mathbf{g}^{\prime}$ is the ultradistributional derivative of $\mathbf{g}$ ) generate the same element in $\mathcal{D}_{L^{1}}^{(s)}(0, T ; E) \cong \mathcal{L}_{b}\left(\dot{\mathcal{B}}^{(s)}(0, T), E\right)$. To see this, for $\varphi \in \dot{\mathcal{B}}^{(s)}(0, T)$, we calculate as follows

$$
\begin{aligned}
\langle\widetilde{\mathbf{f}}, \varphi\rangle & =\sum_{\alpha=0}^{\infty}(-1)^{\alpha} \int_{0}^{T} \widetilde{\mathbf{G}}_{\alpha}^{\prime}(t) \varphi^{(\alpha)}(t) d t \\
& =-\sum_{\alpha=0}^{\infty}(-1)^{\alpha} \int_{0}^{T} \widetilde{\mathbf{G}}_{\alpha}(t) \varphi^{(\alpha+1)}(t) d t,
\end{aligned}
$$

which is exactly the value at $\varphi$ of the ultradistributional derivative of $\mathrm{g} \in \mathcal{D}_{L^{1}}^{(s)}(0, T ; E)$.

We consider the equation

$$
\mathbf{u}^{\prime}=A \mathbf{u}+\mathbf{f} \quad \text { in } \mathcal{D}_{L^{1}}^{(s)}(0, T ; E) .
$$

In other words, $\mathbf{f} \in \mathcal{D}_{L^{1}}^{(s)}(0, T ; E)$ is given, and we search for $\mathbf{u} \in$ $\mathcal{D}_{L^{1}}^{\prime(s)}(0, T ; E)$ such that, for every $\varphi \in \dot{\mathcal{B}}^{(s)}(0, T),\langle\mathbf{u}, \varphi\rangle \in D(A)$ and $\left\langle\mathbf{u}^{\prime}, \varphi\right\rangle=A\langle\mathbf{u}, \varphi\rangle+\langle\mathbf{f}, \varphi\rangle$. By the above discussion, for $1<p<\infty$, there exists $h>0$ and $\mathbf{F}_{\alpha} \in W^{1, p}(0, T ; E), \mathbf{F}_{\alpha}(0)=0, \alpha \in \mathbb{N}$, such that (4.12) holds (with $\mathbf{F}_{\alpha}$ and $\mathbf{F}_{\alpha}^{\prime}$ in place of $\widetilde{\mathbf{G}}_{\alpha}$ and $\widetilde{\mathbf{G}}_{\alpha}^{\prime}$ ) and $\mathbf{f}=\sum_{\alpha=0}^{\infty} \mathbf{F}_{\alpha}^{(\alpha)}$.

If we put $\widetilde{\mathbf{f}}=\left(\mathbf{F}_{\alpha}\right)_{\alpha}$, then $\widetilde{\mathbf{f}} \in \widetilde{\mathcal{D}}_{W^{1, p}, h}^{\prime}(0, T ; E)$. For $u_{0, \alpha}=0 \in$ $D(A)$, put $u_{0}=\left(u_{0, \alpha}\right)_{\alpha}$. Then the conditions of Theorem 4.5 (ii) are satisfied; hence, there exists $\widetilde{\mathbf{u}}=\left(\mathbf{U}_{\alpha}\right)_{\alpha} \in \widetilde{\mathcal{D}}_{W^{1, p}, h}^{\prime}(0, T ; E) \cap$ $\widetilde{\mathcal{D}}_{L^{p}, h}^{\prime s}(0, T ; D(A))$ which is a strict weak solution of $\widetilde{\mathbf{u}}^{\prime}=A \widetilde{\mathbf{u}}+\widetilde{\mathbf{f}}$ in $\widetilde{\mathcal{D}}_{L^{p}, h}^{\prime s}(0, T ; E)$. If we put $\mathbf{u}=\sum_{\alpha=0}^{\infty} \mathbf{U}_{\alpha}^{(\alpha)} \in \mathcal{D}_{L^{1}}^{(s)}(0, T ; E)$, by the above discussion, $\langle\mathbf{u}, \varphi\rangle \in D(A)$, for all $\varphi \in \dot{\mathcal{B}}^{(s)}(0, T)$ (since this holds for $\widetilde{\mathbf{u}})$ and $\mathbf{u}$ is a solution of $\mathbf{u}^{\prime}=A \mathbf{u}+\mathbf{f}$ in $\mathcal{D}_{L^{1}}^{(s)}(0, T ; E)$. Moreover, by Theorem 3.5, this $\mathbf{u}$ as well as $\mathbf{f}$ are in fact elements of $\mathcal{D}_{L^{p}, h}^{(s)}(0, T ; E)$. Thus, we have proved the following strengthened version of Theorem 0.1 (announced in the introduction). Observe that $\mathcal{D}_{L^{1}}^{\prime(s)}(0, T ; E) \cong \mathcal{D}_{L^{p}}^{\prime(s)}(0, T ; E)$, for $1 \leq p \leq \infty$, by the results obtained in the previous sections. 
Theorem 4.6. Let $A: D(A) \subseteq E \rightarrow E$ be a closed operator which satisfies the Hille-Yosida condition and $\mathbf{f} \in \mathcal{D}_{L^{1}}^{(s)}(0, T ; E)$. Then the equation $\mathbf{u}^{\prime}=A \mathbf{u}+\mathbf{f}$ always has a solution $\mathbf{u} \in \mathcal{D}_{L^{1}}^{(s)}(0, T ; E)$. Moreover, $\mathbf{u} \in \mathcal{D}_{L^{p}, h}^{(s)}(0, T ; E)$ where $1<p<\infty$ and $h>0$ are such that

$$
\sum_{\alpha=0}^{\infty} \frac{\alpha !^{p s}}{h^{p \alpha}}\left(\left\|\mathbf{F}_{\alpha}\right\|_{L^{p}(0, T ; E)}^{p}+\left\|\mathbf{F}_{\alpha}^{\prime}\right\|_{L^{p}(0, T ; E)}^{p}\right)<\infty,
$$

with $\mathbf{f}=\sum_{\alpha} \mathbf{F}_{\alpha}^{(\alpha)}$, where $\mathbf{F}_{\alpha} \in W^{1, p}(0, T ; E), \mathbf{F}_{\alpha}(0)=0, \alpha \in \mathbb{N}$.

5. Applications. Theorem 4.6 is applicable in a variety of different situations. We collect some of them in the next proposition. First we need the following definition given in [22].

Definition 5.1. Let $\Omega$ be a bounded open domain with smooth boundary in $\mathbb{R}^{d}$ and $m \in \mathbb{Z}_{+}$. We say that $A\left(x, \partial_{x}\right)=\sum_{|\alpha| \leq 2 m} a_{\alpha}(x) \partial_{x}^{\alpha}$ where $a_{\alpha} \in \mathcal{C}^{2 m}(\bar{\Omega})$ is strongly elliptic if there exists $c>0$ such that

$$
\operatorname{Re}(-1)^{m} \sum_{|\alpha|=2 m} a_{\alpha}(x) \xi^{\alpha} \geq c|\xi|^{2 m}, \quad \text { for all } x \in \bar{U} \text {, for all } \xi \in \mathbb{R}^{d} .
$$

Proposition 5.2. The operator $A: D(A) \subseteq E \rightarrow E$ is a closed operator which satisfies the Hille-Yosida condition in each of the following situations:

(i) $E=\mathcal{C}([0,1]), A v=-v^{\prime}, D(A)=\left\{v \in \mathcal{C}^{1}([0,1]) \mid v(0)=0\right\}$ ([6]).

(ii) For $\kappa \in(0,1), E=\mathcal{C}_{0}^{\kappa}([0,1])=\left\{v \in \mathcal{C}^{\kappa}([0,1]) \mid v(0)=0\right\}$, $A v=-v^{\prime}, D(A)=\left\{v \in \mathcal{C}^{1+\kappa}([0,1]) \mid v(0)=v^{\prime}(0)=0\right\}([\mathbf{6}])$.

(iii) $E=\mathcal{C}([0,1]), A v=v^{\prime \prime}, D(A)=\left\{v \in \mathcal{C}^{2}([0,1]) \mid v(0)=v(1)=0\right\}$ $([6])$.

(iv) For $\Omega$ a bounded open set with regular boundary in $\mathbb{R}^{d}, E=\mathcal{C}(\bar{\Omega})$, $A v=\Delta v, D(A)=\left\{v \in \mathcal{C}(\bar{\Omega}) \mid v_{\mid \partial \Omega}=0, \Delta v \in \mathcal{C}(\bar{\Omega})\right\}$ (here $\Delta$ is the Laplacian in the sense of distributions in $\Omega)([\mathbf{6}])$.

(v) Let $\Omega$ be a bounded open domain with smooth boundary in $\mathbb{R}^{d}$ and $m \in \mathbb{Z}_{+}$. Let $A\left(x, \partial_{x}\right)$ be strongly elliptic. Define $E=L^{p}(\Omega)$, $A v=-A\left(x, \partial_{x}\right) v, D(A)=W^{2 m, p}(\Omega) \cap W_{0}^{m, p}(\Omega)$, for $1<p<\infty$ and for $p=1$ define $E=L^{1}(\Omega), A v=-A\left(x, \partial_{x}\right) v, D(A)=\{v \in$ $\left.W^{2 m-1,1}(\Omega) \cap W_{0}^{m, 1}(\Omega) \mid A\left(x, \partial_{x}\right) v \in L^{1}(\Omega)\right\}([\mathbf{2 2}])$. 
In particular, for $\mathbf{f} \in \mathcal{D}_{L^{1}}^{(s)}(0, T ; E)$, the equation $\mathbf{u}_{t}^{\prime}=A \mathbf{u}+\mathbf{f}$ always has a solution in $\mathcal{D}_{L^{1}}^{(s)}(0, T ; E)$.

Note that the operator $A$ in (v) has a dense domain.

Proof. The fact that $A: D(A) \subseteq E \rightarrow E$ is a closed operator which satisfies the Hille-Yosida condition when $A$ and $E$ are defined as in (i)-(iv) is proven in [6, Section 14]. When $A$ and $E$ are defined as in [22, Theorem 7.3.5 v), page 214] for the case $1<p<\infty$, respectively [22, Theorem 7.3.10, page 218] for the case $p=1$, implies that $A$ is a closed operator which satisfies the Hille-Yosida condition (in fact these theorems state that $A$ is the infinitesimal generator of an analytic semigroup on $\left.L^{p}(\Omega), 1 \leq p<\infty\right)$. Now, the fact that the equation $\mathbf{u}_{t}^{\prime}=A \mathbf{u}+\mathbf{f}$ has a solution in $\mathcal{D}_{L^{1}}^{(s)}(0, T ; E)$ follows from Theorem 4.6.

5.1. Parabolic equation in $\mathcal{D}_{L^{1}}^{(s)}(U)$. In this subsection, $U$ is a bounded domain in $\mathbb{R}^{d}$ with smooth boundary. For the brevity of notation, let $\widetilde{\mathcal{D}}_{L^{p}, h}^{\prime s}(U)$, respectively $\widetilde{\mathcal{D}}_{W^{1, p}, h}^{\prime s}(U)$, be the space $\widetilde{\mathcal{D}}_{L^{p}, h}^{\prime s}(0, T ; E)$, respectively $\widetilde{\mathcal{D}}_{W^{1, p}, h}^{\prime s}(0, T ; E)$, when $E=\mathbb{C}$. Also, for $k \in \mathbb{Z}_{+}$, by $\widetilde{\mathcal{D}}_{W^{k, p}, h}^{\prime s}(U)$, we denote the space of all sequences $\left(F_{\alpha}\right)_{\alpha}$, $F_{\alpha} \in W^{k, p}(U)$, for all $\alpha \in \mathbb{N}^{d}$, for which

$$
\left\|\left(F_{\alpha}\right)_{\alpha}\right\|_{\tilde{\mathcal{D}}_{W^{k, p}, h}^{\prime s}(U)}=\left(\sum_{\alpha \in \mathbb{N}^{d}} \frac{\alpha !^{p s}}{h^{p \alpha}}\left\|F_{\alpha}\right\|_{W^{k, p}(U)}^{p}\right)^{1 / p}<\infty .
$$

It is easy to verify that it becomes a $(B)$-space with the norm $\|\cdot\|_{\widetilde{\mathcal{D}}_{W^{k, p}, h}^{\prime s}(U)} \cdot$

Let $m \in \mathbb{Z}_{+}$,

$$
A\left(x, \partial_{x}\right)=\sum_{|\alpha| \leq 2 m} a_{\alpha}(x) \partial_{x}^{\alpha}
$$

where $a_{\alpha} \in \mathcal{E}^{(s)}(V)$ for some open set $V \subseteq \mathbb{R}^{d}$ and $U \subset \subset V$. We assume that $A\left(x, \partial_{x}\right)$ is a strongly elliptic operator. Obviously, $A\left(x, \partial_{x}\right)$ is a continuous operator on $\dot{\mathcal{B}}^{(s)}(U)$ and on $\mathcal{D}_{L^{1}}^{(s)}(U)$. Denote by 
$\widetilde{A}: D(\widetilde{A}) \subseteq L^{2}(U) \rightarrow L^{2}(U)$ the following unbounded operator

$$
D(\widetilde{A})=W^{2 m, 2}(U) \cap W_{0}^{m, 2}(U), \quad \widetilde{A}(\varphi)=A\left(x, \partial_{x}\right) \varphi, \quad \varphi \in D(\widetilde{A}) .
$$

For such $A\left(x, \partial_{x}\right)$ the following a priori estimate holds (see [22, Theorem 7.3.1, page 212]).

Proposition 5.3. [22]. Let $A\left(x, \partial_{x}\right)$ be a strongly elliptic operator of order $2 m$ on a bounded domain $U$ with smooth boundary $\partial U$ in $\mathbb{R}^{d}$, and let $1<p<\infty$. Then, there exists a constant $\widetilde{C}>0$ such that

$$
\begin{gathered}
\|\varphi\|_{W^{2 m, p}(U)} \leq \widetilde{C}\left(\left\|A\left(x, \partial_{x}\right) \varphi\right\|_{L^{p}(U)}+\|\varphi\|_{L^{p}(U)}\right), \\
\text { for all } \varphi \in W^{2 m, p}(U) \cap W_{0}^{m, p}(U) .
\end{gathered}
$$

Moreover, [22, Theorem 7.3.5, page 214], yields that $-\widetilde{A}$ is the infinitesimal generator of an analytic semigroup of operators on $L^{2}(U)$. In particular, $-\widetilde{A}$ is closed, and it satisfies the Hille-Yosida condition (4.2) for some $\omega, C>0$.

Now we can prove the theorem announced in the introduction. Note that we need to prove the theorem for $\mathcal{D}_{L^{1}}^{(s)}((0, T) \times U)$ since $\mathcal{D}_{L^{p}}^{\prime(s)}((0, T) \times U)$ and $\mathcal{D}_{L^{1}}^{\prime(s)}((0, T) \times U)$ are isomorphic lcs.

Theorem 5.4. Let $U$ be a bounded domain in $\mathbb{R}^{d}$ with smooth boundary, (i.e., $\mathcal{C}^{\infty}$ boundary) and $A\left(x, \partial_{x}\right)$ a strongly elliptic operator of order $2 m$ on $U$. Then, for each $f \in \mathcal{D}_{L^{1}}^{\prime(s)}((0, T) \times U)$ there exists $u \in \mathcal{D}_{L^{1}}^{\prime(s)}((0, T) \times U)$ such that $u_{t}^{\prime}+A\left(x, \partial_{x}\right) u=f$ in $\mathcal{D}_{L^{1}}^{(s)}((0, T) \times U)$.

Proof. Denote by $A$ the following unbounded operator:

$$
\begin{aligned}
A \widetilde{f} & =\left(-A\left(x, \partial_{x}\right) F_{\alpha}\right)_{\alpha}\left(=\left(-\widetilde{A} F_{\alpha}\right)_{\alpha}\right), \\
D(A) & =\left\{\widetilde{f}=\left(F_{\alpha}\right)_{\alpha} \in \widetilde{\mathcal{D}}_{W^{2 m, 2}, h}^{\prime s}(U) \mid F_{\alpha} \in W_{0}^{m, 2}(U), \text { for all } \alpha \in \mathbb{N}^{d}\right\} .
\end{aligned}
$$

Then, obviously, $A: D(A) \subseteq \widetilde{\mathcal{D}}_{L^{2}, h}^{\prime s}(U) \rightarrow \widetilde{\mathcal{D}}_{L^{2}, h}^{\prime s}(U)$ is a linear operator. Since $\widetilde{A}$ is closed, by Proposition 5.3 , it is easy to verify that $A$ is closed. For $\lambda>\omega$, define $B_{\lambda}: \widetilde{\mathcal{D}}_{L^{2}, h}^{\prime s}(U) \rightarrow \widetilde{\mathcal{D}}_{L^{2}, h}^{\prime s}(U)$, by 


$$
\begin{gathered}
B_{\lambda}(\widetilde{f})=\left(R(\lambda:-\widetilde{A}) F_{\alpha}\right)_{\alpha} . \text { For } \widetilde{f}=\left(F_{\alpha}\right)_{\alpha} \in \widetilde{\mathcal{D}}_{L^{2}, h}^{\prime s}(U), \\
\left\|B_{\lambda} \widetilde{f}\right\|_{\widetilde{\mathcal{D}}_{L^{2}, h}^{\prime s}(U)}=\left(\sum_{|\alpha|=0}^{\infty} \frac{\alpha !^{2 s}}{h^{2|\alpha|}}\left\|R(\lambda:-\widetilde{A}) F_{\alpha}\right\|_{L^{2}(U)}^{2}\right)^{1 / 2} \\
\leq \frac{C}{\lambda-\omega}\|\widetilde{f}\|_{\tilde{\mathcal{D}}_{L^{2}, h}^{\prime s}(U)} .
\end{gathered}
$$

Hence, $B_{\lambda}$ is a well-defined continuous operator. For $\left(F_{\alpha}\right)_{\alpha} \in \widetilde{\mathcal{D}}_{L^{2}, h}^{s}(U)$, by the Hille-Yosida condition for $-\widetilde{A}$, Proposition 5.3 and the fact that $\widetilde{A} R(\lambda:-\widetilde{A})=\mathrm{Id}-\lambda R(\lambda:-\widetilde{A})$, we obtain

$$
\left\|R(\lambda:-\widetilde{A}) F_{\alpha}\right\|_{W^{2 m, 2}(U)} \leq \widetilde{C}\left(1+\frac{C(\lambda+1)}{\lambda-\omega}\right)\left\|F_{\alpha}\right\|_{L^{2}(U)} .
$$

This implies that

$$
B_{\lambda}\left(F_{\alpha}\right)_{\alpha}=\left(R(\lambda:-\widetilde{A}) F_{\alpha}\right)_{\alpha} \in \widetilde{\mathcal{D}}_{W^{2 m, 2}, h}^{s}(U) .
$$

Obviously,

$$
R(\lambda:-\widetilde{A}) F_{\alpha} \in W_{0}^{m, 2}(U), \quad \text { for each } \alpha \in \mathbb{N}^{d} .
$$

Hence, the image of $B_{\lambda}$ is contained in $D(A)$. Conversely, for $\left(F_{\alpha}\right)_{\alpha} \in$ $D(A)$, let $G_{\alpha}=(\lambda+\widetilde{A}) F_{\alpha}$, for each $\alpha \in \mathbb{N}^{d}$. Then

$$
\left(G_{\alpha}\right)_{\alpha} \in \widetilde{\mathcal{D}}_{L^{2}, h}^{\prime s}(U)
$$

and

$$
B_{\lambda}\left(G_{\alpha}\right)_{\alpha}=\left(F_{\alpha}\right)_{\alpha} ;
$$

hence, the image of $B_{\lambda}$ is $D(A)$. Also, $(\lambda-A) B_{\lambda}=\operatorname{Id}$ and $B_{\lambda}(\lambda-A)=$ Id. We obtain that $\lambda>\omega$ is in the resolvent of $A, R(\lambda: A)=B_{\lambda}$, and similarly as above, one can prove that

$$
\left\|(\lambda-\omega)^{k} R(\lambda: A)^{k}\right\|_{\mathcal{L}\left(\widetilde{\mathcal{D}}_{L^{\prime}, h}^{s}(U)\right)} \leq C,
$$

i.e., $A$ satisfies the Hille-Yosida condition.

We want to solve the equation $u_{t}^{\prime}(t, x)+A\left(x, \partial_{x}\right) u(t, x)=f(t, x)$ in $\mathcal{D}_{L^{1}}^{\prime(s)}((0, T) \times U)$. For simplicity of notation, put $U_{1}=(0, T) \times U$. By Proposition 2.4, there exist $h>0$ and $F_{\alpha, \beta}(t, x) \in \mathcal{C}\left(\overline{U_{1}}\right), \alpha \in \mathbb{N}$, 
$\beta \in \mathbb{N}^{d}$ such that

$$
f=\sum_{\alpha, \beta} \partial_{t}^{\alpha} \partial_{x}^{\beta} F_{\alpha, \beta} \quad \text { and } \quad \sum_{\alpha, \beta} \frac{(\alpha ! \beta !)^{2 s}}{h^{2(\alpha+|\beta|)}}\left\|F_{\alpha, \beta}\right\|_{L^{\infty}\left(\overline{U_{1}}\right)}^{2}<\infty .
$$

Let $E=\widetilde{\mathcal{D}}_{L^{2}, h}^{\prime s}(U)$. Let $C_{1}^{\prime}=1+\sup _{\beta \in \mathbb{N}^{d}} h^{|\beta|} / \beta !^{s}$, and put $C_{1}=$ $(1+T+|U|) C_{1}^{\prime}$. Let $L_{f}$ be the mapping $\varphi \mapsto L_{f}(\varphi), \dot{\mathcal{B}}^{(s)}(0, T) \rightarrow E$ defined by $L_{f}(\varphi)=\left(\widetilde{F}_{\varphi, \beta}\right)_{\beta}$, where

$$
\widetilde{F}_{\varphi, \beta}(x)=\sum_{\alpha}(-1)^{\alpha} \int_{0}^{T} F_{\alpha, \beta}(t, x) \varphi^{(\alpha)}(t) d t .
$$

We prove that it is a well defined and continuous mapping. First, we prove that $\widetilde{F}_{\varphi, \beta}$ is a continuous function on $\bar{U}$ for each $\beta \in \mathbb{N}^{d}$ and $\varphi \in \dot{\mathcal{B}}^{(s)}(0, T)$. For $\varepsilon>0$, by (5.1), we can find $k_{0} \in \mathbb{Z}_{+}$such that

$$
\sum_{\alpha+|\beta| \geq k_{0}} \frac{(\alpha ! \beta !)^{2 s}}{h^{2(\alpha+|\beta|)}}\left\|F_{\alpha, \beta}\right\|_{L^{\infty}\left(\overline{U_{1}}\right)}^{2}<\frac{\varepsilon^{2}}{\left(4 C_{1}\right)^{2}} .
$$

For each $\alpha \in \mathbb{N}, \beta \in \mathbb{N}^{d}, F_{\alpha, \beta}$ is uniformly continuous (since $\overline{U_{1}}$ is compact in $\mathbb{R}^{d+1}$ ); hence, there exists a $\delta>0$ such that, for every $t, t^{\prime} \in[0, T], x, x^{\prime} \in \bar{U}$ such that $\left|t-t^{\prime}\right| \leq \delta$ and $\left|x-x^{\prime}\right| \leq \delta$,

$$
\sum_{\alpha+|\beta|=0}^{k_{0}-1} \frac{(\alpha ! \beta !)^{2 s}}{h^{2(\alpha+|\beta|)}}\left|F_{\alpha, \beta}(t, x)-F_{\alpha, \beta}\left(t^{\prime}, x^{\prime}\right)\right|^{2}<\frac{\varepsilon^{2}}{\left(2 C_{1}\right)^{2}} .
$$

Hence,

$$
\begin{aligned}
& \left|\widetilde{F}_{\varphi, \beta}(x)-\widetilde{F}_{\varphi, \beta}\left(x^{\prime}\right)\right| \\
& \leq\|\varphi\|_{\mathcal{D}_{L^{2}, h}^{(s)}(0, T)}\left(\sum_{\alpha=0}^{\infty} \frac{(\alpha !)^{2 s}}{h^{2 \alpha}} \int_{0}^{T}\left|F_{\alpha, \beta}(t, x)-F_{\alpha, \beta}\left(t, x^{\prime}\right)\right|^{2} d t\right)^{1 / 2} \\
& \leq \varepsilon\|\varphi\|_{\mathcal{D}_{L^{2}, h}^{(s)}(0, T)},
\end{aligned}
$$


and the continuity of $\widetilde{F}_{\varphi, \beta}$ follows. Also, one easily verifies that

$$
\begin{aligned}
\left(\sum_{\beta} \frac{\beta !^{2 s}}{h^{2|\beta|}}\left\|\widetilde{F}_{\varphi, \beta}\right\|_{L^{\infty}(U)}^{2}\right)^{1 / 2} & \\
& \leq T^{1 / 2}\|\varphi\|_{\mathcal{D}_{L^{2}, h}^{(s)}(U)}\left(\sum_{\alpha, \beta} \frac{(\alpha ! \beta !)^{2 s}}{h^{2(\alpha+|\beta|)}}\left\|F_{\alpha, \beta}\right\|_{L^{\infty}\left(\overline{U_{1}}\right)}^{2}\right)^{1 / 2} .
\end{aligned}
$$

Since

$$
\left\|\widetilde{F}_{\varphi, \beta}\right\|_{L^{2}(U)} \leq|U|^{1 / 2}\left\|\widetilde{F}_{\varphi, \beta}\right\|_{L^{\infty}(U)},
$$

we obtain that $L_{f}$ is well defined and $L_{f} \in \mathcal{L}\left(\dot{\mathcal{B}}^{(s)}(0, T), E\right)$. Now, as $\mathcal{L}_{b}\left(\dot{\mathcal{B}}^{(s)}(0, T), E\right) \cong \mathcal{D}_{L^{1}}^{(s)}(0, T ; E)$ denote by $\mathbf{f} \in \mathcal{D}_{L^{1}}^{(s)}(0, T ; E)$ the mapping $L_{f}$.

Now, Theorem 4.6 implies that there exists $\mathbf{u} \in \mathcal{D}_{L^{1}}^{(s)}(0, T ; E)$ such that $\mathbf{u}^{\prime}=A \mathbf{u}+\mathbf{f}$ in $\mathcal{D}_{L^{1}}^{(s)}(0, T ; E)$. Each element $\mathbf{g}=\left(G_{\alpha}\right)_{\alpha} \in$ $E=\widetilde{\mathcal{D}}_{L^{p}, h}^{\prime s}(U)$ generates an element of $\mathcal{L}_{b}\left(\dot{\mathcal{B}}^{(s)}(U), \mathbb{C}\right)=\mathcal{D}_{L^{1}}^{\prime(s)}(U)$ (see Section 4 ) by

$$
\langle S(\mathbf{g}), \psi\rangle=\sum_{\beta}(-1)^{|\beta|} \int_{U} G_{\beta}(x) \partial_{x}^{\beta} \psi(x) d x,
$$

and one easily verifies that the mapping $S: E \rightarrow \mathcal{D}_{L^{1}}^{(s)}(U), \mathbf{g} \mapsto S(\mathbf{g})$, is continuous. Hence, we have the continuous mapping $\varphi \mapsto S(\langle\mathbf{u}, \varphi\rangle)$, given by

$$
\dot{\mathcal{B}}^{(s)}(0, T) \stackrel{\langle\mathbf{u}, \cdot\rangle}{\longrightarrow} E \stackrel{S}{\rightarrow} \mathcal{D}_{L^{1}}^{(s)}(U) .
$$

Since $\varphi \mapsto S(\langle\mathbf{u}, \varphi\rangle) \in \mathcal{L}_{b}\left(\dot{\mathcal{B}}^{(s)}(0, T), \mathcal{D}_{L^{1}}^{(s)}(U)\right) \cong \mathcal{D}_{L^{1}}^{(s)}\left(U_{1}\right)$ (where the isomorphism follows from Theorem 3.1), denote by $u \in \mathcal{D}_{L^{1}}^{(s)}\left(U_{1}\right)$ this ultradistribution. Then, for $\varphi \in \dot{\mathcal{B}}^{(s)}(0, T), \psi \in \dot{\mathcal{B}}^{(s)}(U)$, $\langle u(t, x), \varphi(t) \psi(x)\rangle=\langle S(\langle\mathbf{u}, \varphi\rangle), \psi\rangle$. Since $\left\langle\mathbf{u}^{\prime}, \varphi\right\rangle=-\left\langle\mathbf{u}, \varphi^{\prime}\right\rangle$, for all $\varphi \in \dot{\mathcal{B}}^{(s)}(0, T)$, we have

$$
\left\langle u_{t}^{\prime}(t, x), \varphi(t) \psi(x)\right\rangle=-\left\langle u(t, x), \varphi^{\prime}(t) \psi(x)\right\rangle=\left\langle S\left(\left\langle\mathbf{u}^{\prime}, \varphi\right\rangle\right), \psi\right\rangle,
$$

for all $\varphi \in \dot{\mathcal{B}}^{(s)}(0, T), \psi \in \dot{\mathcal{B}}^{(s)}(U)$. Also, for $\varphi \in \dot{\mathcal{B}}^{(s)}(0, T)$, since $\langle\mathbf{u}, \varphi\rangle \in D(A)$,

$$
\langle\mathbf{u}, \varphi\rangle=\left(G_{\varphi, \beta}\right)_{\beta} \in D(A) .
$$


Then, by the definition of $A, A\langle\mathbf{u}, \varphi\rangle=\left(-\widetilde{A} G_{\varphi, \beta}\right)_{\beta} \in E$. Now, for $\psi \in \dot{\mathcal{B}}^{(s)}(U)$,

$$
\begin{aligned}
& \left\langle S\left(\left(-\widetilde{A} G_{\varphi, \beta}\right)_{\beta}\right), \psi\right\rangle \\
& =-\sum_{\beta}(-1)^{|\beta|} \int_{U} \widetilde{A} G_{\varphi, \beta}(x) \partial_{x}^{\beta} \psi(x) d x \\
& =-\sum_{\beta}(-1)^{|\beta|} \int_{U} G_{\varphi, \beta}(x)^{t} A\left(x, \partial_{x}\right) \partial_{x}^{\beta} \psi(x) d x \\
& =-\left\langle S(\langle\mathbf{u}, \varphi\rangle),{ }^{t} A\left(x, \partial_{x}\right) \psi\right\rangle \\
& =-\left\langle u(t, x), \varphi(t)^{t} A\left(x, \partial_{x}\right) \psi(x)\right\rangle \\
& =-\left\langle A\left(x, \partial_{x}\right) u(t, x), \varphi(t) \psi(x)\right\rangle,
\end{aligned}
$$

i.e.,

$$
\langle S(A\langle\mathbf{u}, \varphi\rangle), \psi\rangle=-\left\langle A\left(x, \partial_{x}\right) u(t, x), \varphi(t) \psi(x)\right\rangle
$$

for all $\varphi \in \dot{\mathcal{B}}^{(s)}(0, T)$ and $\psi \in \dot{\mathcal{B}}^{(s)}(U)$. Moreover, observe that, for $\varphi \in \dot{\mathcal{B}}^{(s)}(0, T)$ and $\psi \in \dot{\mathcal{B}}^{(s)}(U)$, we have

$$
\begin{aligned}
\langle S(\langle\mathbf{f}, \varphi\rangle), \psi\rangle & =\sum_{\beta}(-1)^{|\beta|} \int_{U} \widetilde{F}_{\varphi, \beta}(x) \partial_{x}^{\beta} \psi(x) d x \\
& =\sum_{\alpha, \beta}(-1)^{\alpha+|\beta|} \int_{U_{1}} F_{\alpha, \beta}(t, x) \varphi^{(\alpha)}(t) \partial_{x}^{\beta} \psi(x) d t d x \\
& =\langle f(t, x), \varphi(t) \psi(x)\rangle,
\end{aligned}
$$

where, in the second equality, we used the definition of $\widetilde{F}_{\varphi, \beta}$ and Fubini's theorem since

$$
\sum_{\alpha, \beta} \int_{U_{1}}\left|F_{\alpha, \beta}(t, x)\right|\left|\varphi^{(\alpha)}(t)\right|\left|\psi^{(\beta)}(x)\right| d t d x<\infty
$$

by (5.1). Now, since $\mathbf{u}^{\prime}=A \mathbf{u}+\mathbf{f}$ in $\mathcal{D}_{L^{1}}^{(s)}(0, T ; E)$, for every $\varphi \in$ $\dot{\mathcal{B}}^{(s)}(0, T),\left\langle\mathbf{u}^{\prime}(t), \varphi(t)\right\rangle=A\langle\mathbf{u}(t), \varphi(t)\rangle+\langle\mathbf{f}(t), \varphi(t)\rangle$ in $E$. Then,

$$
S\left(\left\langle\mathbf{u}^{\prime}, \varphi\right\rangle\right)=S(A\langle\mathbf{u}, \varphi\rangle)+S(\langle\mathbf{f}, \varphi\rangle)
$$

in $\mathcal{D}_{L^{1}}^{(s)}(U)$. Hence, for $\varphi \in \dot{\mathcal{B}}^{(s)}(0, T)$ and $\psi \in \dot{\mathcal{B}}^{(s)}(U)$, we have $\left\langle u_{t}^{\prime}(t, x), \varphi(t) \psi(x)\right\rangle=\left\langle S\left(\left\langle\mathbf{u}^{\prime}, \varphi\right\rangle\right), \psi\right\rangle$ 


$$
\begin{aligned}
& =\langle S(A\langle\mathbf{u}, \varphi\rangle), \psi\rangle+\langle S(\langle\mathbf{f}, \varphi\rangle), \psi\rangle \\
& =-\left\langle A\left(x, \partial_{x}\right) u(t, x), \varphi(t) \psi(x)\right\rangle+\langle f(t, x), \varphi(t) \psi(x)\rangle .
\end{aligned}
$$

Since $\dot{\mathcal{B}}^{(s)}(0, T) \widehat{\otimes} \dot{\mathcal{B}}^{(s)}(U) \cong \dot{\mathcal{B}}^{(s)}\left(U_{1}\right)$ by Theorem 3.1, we obtain the claim in the theorem.

Example 5.5. An interesting application of this theorem is obtained by taking $A\left(x, \partial_{x}\right)$ to be $-\Delta_{x}\left(\Delta_{x}\right.$ is the Laplacian $\left.\partial_{x_{1}}^{2}+\cdots+\partial_{x_{d}}^{2}\right)$ and $U$ to be the arbitrary bounded domain with smooth boundary in $\mathbb{R}^{d}$. Then $-\Delta_{x}$ is a strongly elliptic operator of order 2 on $U$. The above theorem then asserts that, for $f \in \mathcal{D}_{L^{1}}^{\prime(s)}((0, T) \times U)$, the equation $u_{t}^{\prime}-\Delta_{x} u=f$ always has a solution in $\mathcal{D}_{L^{1}}^{\prime(s)}((0, T) \times U)$.

Example 5.6. If $U=\left(0, T_{1}\right) \subseteq \mathbb{R}$ and $A$ is the differentiation in $x$, arguing as above, one can prove the following assertion:

Let $f \in \mathcal{D}_{L^{1}}^{\prime(s)}\left((0, T) \times\left(0, T_{1}\right)\right)$. The equation $u_{t}^{\prime}+u_{x}^{\prime}=f$ always has a solution in $\mathcal{D}_{L^{1}}^{(s)}\left((0, T) \times\left(0, T_{1}\right)\right)$.

\section{REFERENCES}

1. W. Arendt, Vector-valued Laplace transforms and Cauchy problems, Israel J. Math. 59 (1987), 327-352.

2. W. Arendt, C.J.K. Batty, M. Hieber and F. Neubrander, Vector-valued Laplace transforms and Cauchy problems, Springer, Basel, 2011.

3. R. Beals, On the abstract Cauchy problem, J. Funct. Anal. 10 (1972), 281299 .

4. 300-308.

5. J. Chazarain, Problémes de Cauchy abstraites et applications á quelques problémes mixtes, J. Funct. Anal. 7 (1971), 386-446.

6. G. Da Prato and E. Sinestrari, Differential operators with nondense domain, Ann. Scuola Norm. Sup. Pisa 14 (1987), 285-344.

7. M. Hieber, Integrated semigroups and differential operators on $L^{p}$ spaces, Math. Ann. 29 (1991), 1-16.

8. H. Kellermann and M. Hieber, Inegrated semigroups, J. Funct. Anal. 84, (1989), 160-180.

9. H. Komatsu, Ultradistributions, I. Structure theorems and a characterization, J. Fac. Sci. Univ. Tokyo 20 (1973), 25-105. 
10. H. Komatsu, Ultradistributions, II: The kernel theorem and ultradistributions with support in submanifold, J. Fac. Sci. Univ. Tokyo 24 (1977), 607-628.

11. Ultradistributions, III. Vector valued ultradistributions the theory of kernels, J. Fac. Sci. Univ. Tokyo 29 (1982), 653-718.

12. M. Kostić, Generalized semigroups and cosine functions, Mathematical Institute SANU, Belgrade, 2011.

13. M. Kostić and S. Pilipović, Global convoluted semigroups, Math Nachr. 280 (2007), 1727-1743.

14. UL Ultradistribution semigroups, Siberian Math. J. 53 (2012), 232-242.

15. P.C. Kunstmann, Distribution semigroups and abstract Cauchy problems, Trans. Amer. Math. Soc. 351 (1999), 837-856.

16. P.C. Kunstmann, Banach space valued ultradistributions and applications to abstract Cauchy problems, http://math.kit.edu/iana1/ kunstmann/ media/ultra-appl.pdf.

17. R. de Laubenfels and F. Yao, Regularized semigroups of bounded semivariation, Semigroup Forum 54 (1997), 43-57.

18. A. Lunardi, Analytic semigroups and optimal regularity in parabolic problem, Birkhäuser, Basel, 1995.

19. I.V. Melnikova and A.I. Filinkov, Abstract Cauchy problems: Three approaches, Chapman \& Hall/CRC, Washington, 2001.

20. R. Nagel and E. Sinestrari, Inhomogeneous Volterra integrodifferential equations for Hille-Yosida operators Dekker Lect. Notes 150 (1994), 51-70, Dekker.

21. F. Neubrander, Integrated semigroups and their applications to the abstract Cauchy problem, Pacific J. Math. 135 (1988), 111-155.

22. A. Pazy, Semigroups of linear operators and applications to partial differential equations, Springer-Verlag, New York, 1983.

23. R. Phillips, Perturbation theory of semigroups of linear operators, Trans. Amer. Math. Soc. 74 (1953), 199-221.

24. S. Pilipović, Characterizations of bounded sets in spaces of ultradistributions, Proc. Amer. Math. Soc. 120 (1994), 1191-1206.

25. E. Sinestrari, On the abstract Cauchy problem of parabolic type in space of continuous functions, J. Math. Anal. Appl. 107 (1985), 16-66.

26. Hille-Yosida operators and Cauchy problems, Semigroup Forum $\mathbf{8 2}$ (2011), 10-34.

27. H. Schaefer, Topological vector spaces, 3rd edition, Springer-Verlag, New York, 1971.

28. L. Schwartz, Théorie des distributions I, Herman, Paris, 1966.

29. 7 (1957), 1-141. 
University of Novi Sad, Trg D. Obradovića 4, 21000 Novi Sad, Serbia

Email address: pilipovic@dmi.uns.ac.rs

Department for Mathematics, Faculty of Mechanical Engineering-Skopje, Karposh 2 B.B., 1000 Skopje, Macedonia

Email address: bprangoski@yahoo.com

Department for Mathematics, Faculty of Civil Engineering-Skopje, PartiZANSki Odredi 24, P.O. Box 560, 1000 Skopje, Macedonia

Email address: velinovd@gf.ukim.edu.mk 